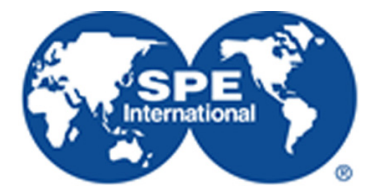

Society of Petroleum Engineers

\title{
SPE-177481-MS
}

\section{Numerical Simulation of Natural Gas Flow in Anisotropic Shale Reservoirs}

Ardiansyah Negara, Baker Hughes; Amgad Salama and Shuyu Sun, King Abdullah University of Science and Technology; Mokhtar Elgassier, Baker Hughes; Yu-Shu Wu, Colorado School of Mines

Copyright 2015, Society of Petroleum Engineers

This paper was prepared for presentation at the Abu Dhabi International Petroleum Exhibition and Conference held in Abu Dhabi, UAE, 9-12 November 2015.

This paper was selected for presentation by an SPE program committee following review of information contained in an abstract submitted by the author(s). Contents of the paper have not been reviewed by the Society of Petroleum Engineers and are subject to correction by the author(s). The material does not necessarily reflect any position of the Society of Petroleum Engineers, its officers, or members. Electronic reproduction, distribution, or storage of any part of this paper without the written consent of the Society of Petroleum Engineers is prohibited. Permission to reproduce in print is restricted to an abstract of not more than 300 words; illustrations may not be copied. The abstract must contain conspicuous acknowledgment of SPE copyright.

\section{Abstract}

Shale gas resources have received great attention in the last decade due to the decline of the conventional gas resources. Unlike conventional gas reservoirs, the gas flow in shale formations involves complex processes with many mechanisms such as Knudsen diffusion, slip flow (Klinkenberg effect), gas adsorption and desorption, strong rock-fluid interaction, etc. Shale formations are characterized by the tiny porosity and extremely low-permeability such that the Darcy equation may no longer be valid. Therefore, the Darcy equation needs to be revised through the permeability factor by introducing the apparent permeability. With respect to the rock formations, several studies have shown the existence of anisotropy in shale reservoirs, which is an essential feature that has been established as a consequence of the different geological processes over long period of time. Anisotropy of hydraulic properties of subsurface rock formations plays a significant role in dictating the direction of fluid flow. The direction of fluid flow is not only dependent on the direction of pressure gradient, but it also depends on the principal directions of anisotropy. Therefore, it is very important to take into consideration anisotropy when modeling gas flow in shale reservoirs. In this work, the gas flow mechanisms as mentioned earlier together with anisotropy are incorporated into the dual-porosity dual-permeability model through the full-tensor apparent permeability. We employ the multipoint flux approximation (MPFA) method to handle the full-tensor apparent permeability. We combine MPFA method with the experimenting pressure field approach, i.e., a newly developed technique that enables us to solve the global problem by breaking it into a multitude of local problems. This approach generates a set of predefined pressure fields in the solution domain in such a way that the undetermined coefficients are calculated from these pressure fields. In other words, the matrix of coefficients is constructed automatically within the solver. We ran a numerical model with different scenarios of anisotropy orientations and compared the results with the isotropic model in order to show the differences between them. We investigated the effect of anisotropy in both the matrix and fracture systems. The numerical results show anisotropy plays a crucial role in dictating the pressure fields as well as the gas flow streamlines. Furthermore, the numerical results clearly show the effects of anisotropy on the production rate and cumulative production. Incorporating anisotropy together with gas flow mechanisms in shale formations into the reservoir model is essential particularly for predicting maximum gas production from shale reservoirs. 


\section{Introduction}

International Energy Agency (IEA 2011) reports that the world's natural gas demand and supply will increase in the future such that the natural gas becomes a key role in the global energy mix. It is expected that global primary demand will reach 5.1 trillion cubic meters (tcm) in 2035, which is 1.8 tcm more than today's consumption. This definitely requires additional gas resources in addition to existing conventional gas recourses in order to improve the energy security. One promising gas resource that has received great attention in the past decade is unconventional gas resources particularly shale gas. According to a published EIA study in 2013, world shale gas resources are about 7,299 tcm (EIA 2013). Even with these very large reserves and the fact that there has been some success in increasing gas production from shale reservoirs, unfortunately, the physicochemical processes that take place in the shale formations remain challenging and are not completely understood. Unlike conventional gas reservoirs, the gas flow in shale formations involves complex flow processes with many different mechanisms act at the same time. These processes include mechanisms such as Knudsen molecular diffusion, gas-slippage effect (Klinkenberg effect), adsorption and desorption, strong rock-fluid interaction, rock deformation, etc (Shi et al. 2013; Guo et al. 2014; Wu et al. 2014). Shale formations are characterized by the very tiny pores and extremely low permeability in which different shale types will have different characteristics although the porosity, stress, and pore pressure are similar (Wu et al. 2014). Experimental results from tests on the core samples indicate that typical permeability of shale formation is 54 nanodarcy (nd). $90 \%$ of the cores are less than $150 \mathrm{nd}$, and the porosity varies from 4 nanometer (nm) to $200 \mathrm{~nm}$ (Javadpour et al. 2007).

Understanding the gas flow process in shale reservoirs is very important. The gas is stored in two different continuum porous media: 1) free gas and adsorbed gas co-existing in the matrix nanopores; and 2) free gas trapped in the micro-fracture porosity. In the nanopores-dominated matrix, the gas is desorbed from the rock surface into the matrix pore network. Then, the gas flows from the nano-matrix to the micro-fracture network due to the pressure gradient between the matrix and fracture systems. Eventually, the gas is produced in the wellbore. A comprehensive understanding with regard to the complex flow processes as mentioned earlier is certainly required to build a correct mathematical model for shale gas flow. In tight formations like shale gas, the traditional Darcy flow becomes invalid because of the nanodarcy matrix permeability (Ozkan et al. 2010). At nanoscale permeability, Knudsen diffusion becomes the dominant mechanism that drives the gas flow from the matrix to the fracture network. Thus, a revised Darcy's law particularly in the permeability factor is needed to take into account several physical mechanisms, e.g., Knudsen diffusion and slip flow (Klinkenberg effect). This leads to the so- called apparent permeability. Unlike conventional reservoirs in which permeability is only a function of the rock systems, the permeability in shale reservoirs depends also on the reservoir pressure (Javadpour 2009; Mehmani et al. 2013).

In addition to the incomplete understanding of the previously mentioned physicochemical mechanisms, there is another challenge to the complexity of the geological structure of the subsurface. Due to geologic processes over long period of time, the rock formations are anisotropic. This means the permeability is dependent on the directions. As a consequence, fluid flow direction depends on both pressure gradient and the principal directions of anisotropy. Several studies have shown the existence of anisotropy in the shale formations (McCarthy 1991; Ayan et al. 1994; Kwon et al. 2004; Burton 2011). In fact, anisotropy exists in the scale of micrometer or nanometer under microstructural observations using transmission electron microscopy (TEM) (Kwon et al. 2004). To take into account such anisotropy into the model, the permeability has to be represented as a full tensor. The standard finite difference of two-point flux approximation (TPFA) is, unfortunately, not capable of handling the full-tensor permeability. This limits the use of this method in many porous media applications. However, a method has been developed to handle full-tensors. This method is called multipoint flux approximation (MPFA). It can handle anisotropy in the framework of finite difference schemes. MPFA method was first introduced by Aavatsmark et al. 
(1998) and Aavatsmark (2002). MPFA method has been widely implemented in many engineering applications (Keilegavlen et al. 2012; Osman et al. 2012; Negara 2015; Negara et al. 2013, 2014, 2015; Salama et al. 2013, 2014, In press). As indicated by its name, MPFA involves more point stencils than TPFA, e.g., 9-point stencil and 27-point stencil for 2-D and 3-D problems, respectively. Having more point stencils will certainly make the construction of the matrix coefficient more complicated. Therefore, we apply the so-called experimenting pressure field approach. This technique enables the solution of a global problem breaks down into solution of multitude of local problems where a set of predefined pressure fields are applied to the solution domain and the undetermined coefficients are calculated. In other words, the matrix of coefficients is constructed automatically within the solver. This approach has been implemented successfully in recent years (Sun et al. 2012; Negara 2015; Negara et al. 2013, 2014, 2015; Salama et al., 2013, 2014, In press).

In light of the above background discussion, this paper is devoted to the discussion of modeling natural gas flow in anisotropic shale reservoirs using the dual-porosity dual-permeability model. We consider the anisotropy exists in the matrix and fracture systems. In this work, a numerical experiment with several scenarios of anisotropy orientations has been considered. A comparison between anisotropic and isotropic cases is also presented. The numerical results show that anisotropy plays a crucial role in dictating the pressure fields as well as the gas flow streamlines. Furthermore, the numerical results emphasize the effects of anisotropy on the production rate and cumulative production.

\section{Shale Gas Modeling Equations}

\section{Gas Adsorption and Desorption}

Gas adsorption and desorption processes in shale reservoirs involve free gas and adsorbed gas. Incorporating such processes into shale gas model is very important to predict the total gas flow or production. When the pore pressure decreases, adsorbed gas is released from the surface of kerogen and becomes free gas. This free gas then flows to production wells through fracture networks. The amount of adsorbed gas is determined by the Langmuir's isotherm model (Langmuir 1916). Langmuir's isotherm model describes the amount of adsorbed gas in shale formations. This depends on the pressure and temperature of the system. This empirical model is analogous to the one used in coalbed methane and based on the assumption that there is an instantaneous equilibrium between the sorbed gas and the free gas (Wu et al. 2014). The Langmuir's isotherm model has been used in much of the literature on shale gas (Cui et al. 2009; Civan et al. 2011; Ding et al. 2014; Guo et al. 2014, Wu et al. 2014; Pan and Connel 2015) and it is given by

$$
q_{a}=\frac{V_{L} p}{p_{L}+p}
$$

which can be expressed as

$$
q=\frac{\rho_{s} M_{g}}{V_{s t d}} q_{a}=\frac{\rho_{s} M_{g}}{V_{s t d}} \frac{V_{L} p}{p_{L}+p}
$$

where $q$ is the mass of gas adsorbed per solid volume $\left(\mathrm{kg} / \mathrm{m}^{3}\right), q_{a}$ is the standard volume of adsorbed gas per solid mass $\left(\mathrm{m}^{3} / \mathrm{kg}\right), \rho_{s}$ is the shale density $\left(\mathrm{kg} / \mathrm{m}^{3}\right), M_{g}$ is the gas molecular weight $(\mathrm{kg} / \mathrm{mol}), V_{\text {std }}$ is the gas molar volume at standard condition $(273.15 \mathrm{~K}, 1 \mathrm{~atm}), V_{L}$ is the Langmuir gas volume $\left(\mathrm{m}^{3} / \mathrm{kg}\right)$, $p$ is the gas pressure $(\mathrm{Pa})$, and $p_{L}$ is the Langmuir gas pressure $(\mathrm{Pa})$. The Langmuir's volume is a function of the organic richness and thermal maturity of shale formation (Wu et al. 2014).

\section{Gas Flow Regime based on Knudsen Number}

In general, the gas flow patterns through microchannels and nanopores are categorized into four flow regimes based on the Knudsen number (Roy 2003). The four flow regimes are continuum, slip, transition 
and free-molecular, as summarized in Table 1. Knudsen number (Knudsen 1909), $K_{n}$, is a dimensionless parameter defined as

Table 1-Gas flow regime based on Knudsen number, $\mathrm{K}_{\mathbf{n}} \cdot$
\begin{tabular}{|ccccc|}
\hline $\mathrm{K}_{\mathbf{n}}$ & $\mathbf{0 - 1 0 ^ { - 3 }}$ & $\mathbf{1 0 ^ { - 3 } - 1 0 ^ { - 1 }}$ & $\mathbf{1 0 ^ { - 1 } - 1 0}$ & $>10$ \\
\hline Flow regime & Continuum & Slip & Transition & Free-molecular \\
\hline & $K_{n}=\frac{\lambda}{D}$ \\
\end{tabular}

where $\lambda$ is the gas mean free path $(\mathrm{m})$ and $D$ is the diameter of the pore $(\mathrm{m})$. According to Heidemann et al. (1984) in Javadpour et al. (2007), the gas mean free path is given by

$$
\lambda=\frac{k_{B} T}{\sqrt{2} \pi d^{2} p}
$$

where $k_{B}$ is the Boltzmann constant $\left(1.38065 \times 10^{-} \mathrm{J} / \mathrm{K}\right), T$ is temperature $(\mathrm{K}), p$ is pressure $(\mathrm{Pa})$, and $d$ is the collision diameter of the gas molecule $(\mathrm{m})$. Knudsen number is a function of pressure for different pore sizes ranging from $10 \mathrm{~nm}$ to $50 \mu \mathrm{m}$ (Javadpour et al. 2007). Looking at Eq. 3 and Eq. 4, we can see that Knudsen number decreases with pressure and pore size. In the continuum flow regime where $K_{n}<$ $10^{-3}$ the gas motion is dominated by the collision between gas molecules without slippage effect. For Knudsen number $10^{-3}<K_{n}<10^{-1}$, the gas flow enters the slip flow regime. In this regime slippage comes into effect (the gas flow at the pore wall is no longer zero). When $10^{-1}<\mathrm{K}_{\mathrm{n}}<10$, the gas flow is in the transitional regime where the motion of the gas is mainly affected by the collisions of the gas molecules as well as the collisions between the gas molecules and the pore wall. The gas flow in the transitional regime is a combination of Knudsen diffusion and slip flow. The last gas flow regime is the free molecule regime, which occurs when $K_{n}>10$. The gas flow in the free molecule regime is dominated mainly by the collisions between the gas molecules and the pore wall. The gas flow in the free molecule regime is only Knudsen diffusion.

\section{Gas Slippage (Klinkenberg) Effect}

In shale gas formations with very low permeability, tiny pores and low pressure condition, the gasslippage effect or Klinkenberg effect (Klinkenberg 1941) may be significant and cannot be ignored in the model. Under such conditions, the permeability is not only a function of topology and morphology of the pores but also a function of the gas pressure, which is expressed as apparent permeability,

$$
\mathbf{K}_{a p p}=\mathbf{K}_{\infty}\left(1+\frac{b}{p}\right)
$$

where $\mathrm{K}_{\infty}$ is the intrinsic or absolute permeability of gas-phase under very large gas-phase pressure (m or $\mathrm{md}), b$ is the slip coefficient $(\mathrm{Pa})$, and $p$ is the pressure $(\mathrm{Pa})$. From Eq. 5 we can see that the apparent permeability is inversely proportional to the pressure, implying that the apparent permeability will increase when the pressure decreases. Therefore, the gas production can be enhanced in low-pressure zone such as near-well region or matrix portions near fractures (Wu et al. 2014). The Klinkenberg effect is expected to be stronger in unconventional reservoirs than conventional reservoirs due to their lowpermeability and tiny pores.

\section{Knudsen Diffusion}

Knudsen diffusion occurs when the gas mean free path is of the same order of magnitude as the diameter of the pore. This implies that the Knudsen flow exists in the free-molecular flow regime and partially in the transition flow regime. The general Knudsen diffusion equation is described as follows: 


$$
J_{K}=-D_{K} \nabla n_{i}
$$

where $J_{K}$ is the mass flux of component- $i\left(\mathrm{~kg} / \mathrm{m}^{3} / \mathrm{s}\right), \mathrm{D}_{\mathrm{K}}$ is Knudsen diffusion coefficient $\left(\mathrm{m}^{2} / \mathrm{s}\right)$, and $\nabla n_{i}$ is the density gradient $\left(\mathrm{kg} / \mathrm{m}^{4}\right)$. Knudsen diffusion coefficient is given by (Roy et al. 2003; Javadpour et al. 2007)

$$
D_{K}=\frac{D}{3} \sqrt{\frac{8 R T}{\pi M_{g}}}
$$

where $D$ is the diameter of the pore $(\mathrm{m}), R$ is the universal gas constant $(8.314 \mathrm{~Pa} \cdot \mathrm{m} /(\mathrm{mol} \cdot \mathrm{K}))$. Knudsen diffusion is significant when pressure is low and pore diameters are small. In the Knudsen diffusion, collision between gas molecules and pore wall is more dominant than the collision between the gas molecules.

\section{Governing Equations}

The governing equations for shale gas modeling involve the mass conservation equation and the revised Darcy's law with the apparent permeability. In this work, we adapt the dual-porosity dual-permeability model for simulating gas flow in shale reservoirs. The dual-porosity dual-permeability model was initially introduced by Barenblatt et al. (1960) and then further developed by others (Warren and Root 1963; Kazemi et al. 1976). Dual-porosity dual-permeability model assumes two different continuum porous media, i.e., matrix and fracture. The matrix is characterized by tiny porosity and very low- permeability and the fracture is characterized by higher porosity and permeability. The governing equations in the dual-porosity dual-permeability model are represented by the two mass conservation equations, one for the matrix and the other for fracture. These equations are:

$$
\begin{aligned}
& \left(\frac{d M}{d t}\right)_{m}+\left(\nabla \cdot\left(\rho_{g} \mathbf{u}\right)\right)_{m}=-T_{m f} \\
& \left(\frac{d M}{d t}\right)_{f}+\left(\nabla \cdot\left(\rho_{g} \mathbf{u}\right)\right)_{f}=T_{m f}-\bar{q}
\end{aligned}
$$

where $M$ is the mass accumulation $\left(\mathrm{kg} / \mathrm{m}^{3}\right), \rho$ is the density $\left(\mathrm{kg} / \mathrm{m}^{3}\right), T_{m f}$ is the transfer term $\left(\mathrm{kg} / \mathrm{m}^{3} / \mathrm{s}\right)$, and $\bar{q}$ is the sink/source $(\mathrm{kg} / \mathrm{m} / \mathrm{s})$. The subscripts $\mathrm{m}$ and $\mathrm{f}$ represent the matrix and fracture, respectively. $\mathbf{u}$ is the revised Darcy velocity given by

$$
\mathbf{u}=-\frac{\mathbf{K}_{a p p}}{\mu_{g}}\left(\nabla p-\rho_{g} \mathbf{g}\right)
$$

\section{Incorporation of Adsorption and Desorption}

The gas adsorption and desorption processes are incorporated in the mass accumulation term. Natural gas is present in the shale formations in two forms, free gas and adsorbed gas. Both forms are present in the matrix. The fracture contains only free gas. According to Guo et al. (2014), the amount of free gas and adsorbed gas is given by

$$
\begin{gathered}
M_{\text {free }}=\phi S_{g} \rho_{g} \\
M_{\text {adsorbed }}=(1-\phi) q
\end{gathered}
$$

where $\phi$ is the porosity of shale, $S_{g}$ is the gas saturation, $\rho_{g}$ is the gas density under standard condition $\left(\mathrm{kg} / \mathrm{m}^{3}\right)$, and $q$ is the mass of gas adsorbed per solid volume $\left(\mathrm{kg} / \mathrm{m}^{3}\right)$ defined by Eq. 2 . Assuming $100 \%$ gas saturation $\left(S_{\mathrm{g}}=1\right)$, the mass accumulation of free gas and adsorbed gas in the matrix is: 


$$
M_{\text {matrix }}=\phi_{m} \rho_{g}+\left(1-\phi_{m}\right) q
$$

And the mass accumulation term for the matrix is:

$$
\left(\frac{d M}{d t}\right)_{m}=\frac{\partial\left(\phi_{m} \rho_{g}\right)}{\partial t}+\frac{\partial\left(\left(1-\phi_{m}\right) q\right)}{\partial t}
$$

Assuming ideal gas behavior.

The density of the gas is:

$$
p V=n R T=\frac{m}{M} R T
$$

$$
\rho_{g}=\frac{m_{g}}{V}=\frac{p M_{g}}{R T}=p \gamma
$$

where $\gamma$ is a coefficient equal to $\underline{M_{g}}$. Substituting Eq. 2 and Eq. 16 into Eq. 14, we obtain the mass accumulation term for the matrix: $\overline{R T}$

$$
\left(\frac{d M}{d t}\right)_{m}=\frac{\partial p_{m}}{\partial t}\left(\phi_{m} \gamma+\frac{\left(1-\phi_{m}\right) \rho_{s} M_{g} p_{L} V_{L}}{V_{s t d}\left(p_{L}+p_{m}\right)^{2}}\right)
$$

In the fracture there is only free gas. Therefore, the mass accumulation term for the fracture is:

$$
\left(\frac{d M}{d t}\right)_{f}=\frac{\partial\left(\phi_{f} \rho_{g}\right)}{\partial t}=\frac{\partial p_{f}}{\partial t}\left(\phi_{f} \gamma\right)
$$

\section{Incorporation of Klinkenberg Effect and Knudsen Diffusion}

The matrix in shale reservoirs is characterized by the tiny porosity and very low-permeability. Consequently, the gas flow mechanism in the matrix is a combination of Klinkenberg effect and Knudsen diffusion. The Klinkenberg effect is when the gas velocity at the pore wall is no longer zero, while Knudsen diffusion occurs when the gas mean free path is close to the size of the pore diameter. These two mechanisms are incorporated in the flow model through the apparent permeability.

Rewriting Eq. 5 for the matrix:

$$
\mathbf{K}_{a p p}=\mathbf{K}_{m}\left(1+\frac{b_{m}}{p_{m}}\right)
$$

here, $\mathrm{K}_{\mathrm{m}}$ is equal to $\mathrm{K}_{\infty}$. The coefficient $b_{m}$ takes into account the Knudsen diffusion and Klinkenberg effect. In this paper, we are using the coefficient of $b_{m}$ as defined by Javadpour (2009):

$$
b_{m}=\frac{16 \mu_{g}}{3000 r}\left(\frac{8 R T}{\pi M_{g}}\right)^{0.5}+\left(\frac{8 \pi R T}{M_{g}}\right)^{0.5} \frac{\mu_{g}}{r}\left(\frac{2}{\beta}-1\right)
$$

where $\mu \mathrm{g}$ is the gas viscosity $(\mathrm{Pa} \cdot \mathrm{s}), r$ is the pore radius $(\mathrm{m})$, and $\beta$ is the tangential momentum accommodation coefficient or part of the gas molecules that is reflected diffusively from the tube wall relative to specular reflection. The two terms of the right side of Eq. 20 are function of reservoir pressure, described by Knudsen number (Beskok and Karniadakis 1999; Michalis et al. 2010),

$$
\mu_{g}=\mu_{g 0} \frac{1}{1+\omega K_{n}}
$$

The Knudsen number is given by (Civan et al. 2011),

$$
K_{n}=\frac{\mu_{g}}{4 p_{m}} \sqrt{\frac{\pi R T \phi_{m}}{M_{g} \tau K_{\infty}}}
$$


here, $\mu_{g 0}$ is the initial gas viscosity $(\mathrm{Pa} \cdot \mathrm{s}), \omega$ is the rarefaction coefficient, and $\tau$ is the tortuosity. The pore radius in Eq. 20 depends on gas adsorption and desorption processes. The more gas adsorbed into the solid (rock) the smaller the pore radius and vice versa. We follow Xiong et al. (2012) to calculate the effective pore radius,

$$
r_{e f f}=r-d_{g} \frac{p_{m} / p_{L}}{1+p_{m} / p_{L}}
$$

where $d_{g}$ is the diameter of the gas molecule $(\mathrm{m})$, e.g., methane $\left(\mathrm{CH}_{4}\right)$.

\section{Final PDE Model}

The mass accumulation terms for both matrix and fracture are given by Eq. 17 and Eq. 18. Substituting the revised Darcy's law Eq. 10 into Eq. 8, we obtain the flow vector term for the matrix (ignoring the gravity for simplification),

$$
(\nabla \cdot(\rho \mathbf{u}))_{m}=-\nabla \cdot\left(\rho_{m}\left(\frac{\mathbf{K}_{a p p, m}}{\mu_{g}} \nabla p_{m}\right)\right)=-\nabla \cdot\left(\gamma p_{m}\left(\frac{\mathbf{K}_{a p p, m}}{\mu_{g}} \nabla p_{m}\right)\right)
$$

Darcy's equation is still valid for modeling gas flow in the fracture network due to its larger pore size and higher permeability, usually in the order of micrometer and millidarcy, respectively. Therefore, the Knudsen diffusion and Klinkenberg effect are not significant in the fracture network.

The flow term for the fracture network is:

$$
(\nabla \cdot(\rho \mathbf{u}))_{f}=-\nabla \cdot\left(\rho_{f}\left(\frac{\mathbf{K}_{f}}{\mu_{g}} \nabla p_{f}\right)\right)=-\nabla \cdot\left(\gamma p_{f}\left(\frac{\mathbf{K}_{f}}{\mu_{g}} \nabla p_{f}\right)\right)
$$

Combining the mass accumulation terms and the flow vector terms results in a set of partial differential equations of the governing equations for shale gas modeling. These equations are:

$$
\begin{gathered}
\left(\phi_{m} \gamma+\frac{\left(1-\phi_{m}\right) M_{g} p_{L} V_{L} \rho_{s}}{V_{s t d}\left(p_{L}+p_{m}\right)^{2}}\right) \frac{\partial p_{m}}{\partial t}+\nabla \cdot\left(-\gamma\left(\frac{\mathbf{K}_{m}\left(p_{m}+b_{m}\right)}{\mu_{g}} \nabla p_{m}\right)\right)=-T_{m f} \\
\phi_{f} \gamma \frac{\partial p_{f}}{\partial t}+\nabla \cdot\left(-\gamma p_{f}\left(\frac{\mathbf{K}_{f}}{\mu_{g}} \nabla p_{f}\right)\right)=T_{m f}+\bar{q}
\end{gathered}
$$

where $p_{m}$ and $P_{f}$ are the pressures of the matrix and fracture, respectively, and $T_{m f}$ is the transfer term that describes the exchange of gas between the matrix and fracture. The negative sign in Eq. 8 indicates a sink term, which implies that the fluid is flowing out from the matrix. There are two approaches to define the transfer term: 1) Warren-Root approach (Warren and Root 1963) and 2) boundary condition approach (Pirson, 1953). The first approach is based on the matrix shape factors (Warren and Root 1963; Kazemi et al. 1976). The Warren-Root approach assumes the fluid transfer from the matrix to the fracture network occurs at pseudo-steady state condition when the no-flow boundary condition is imposed. The transfer term in the first approach is directly related to the shape factor, density of the fluid, viscous force, and potential difference (pressure gradient) at the interface between the matrix and fracture. Meanwhile, the boundary condition approach calculates the cross flux between the matrix and fracture systems explicitly by imposing boundary condition in each time step. This approach is useful for well testing, however, inefficient for large-scale simulation due to high computational cost (Guo et al. 2014). In this work, we only discuss the Warren-Root approach and the transfer term in this approach is represented by

$$
T_{m f}=\frac{K_{m} \rho_{g} \sigma\left(p_{m}-p_{f}\right)}{\mu_{g}}
$$


where $K_{m}$ is the effective permeability of the matrix $\left(\mathrm{m}^{2}\right.$ or md) and $\sigma=4\left(\frac{1}{L_{x}^{2}}+\frac{1}{L_{y}^{2}}+\frac{1}{L_{z}^{2}}\right)$ is the shape factor or the crossflow coefficient between matrix and fracture, $L_{x}, L_{y}$, and $L_{z}$ are the fracture spacing in the $x, y$, and $z$ directions, respectively.

\section{Numerical Method}

\section{Multipoint Flux Approximation}

In this section, we discuss the fundamental concept of multipoint flux approximation (MPFA) and its numerical discretization based on the framework of finite difference. MPFA has been introduced in the late nineties by Edwards and Rogers (1998), Aavatsmark et al. (1998), and Aavatsmark (2002). MPFA is designed to provide better discretization of the flow equation when the grid directions are not aligned with the principal directions of permeability tensor. In other words, MPFA is designed for general orientations of the principal directions of the permeability tensor. This capability can capture the anisotropy phenomena that occur in the subsurface formations, which cannot be handled by two-point flux approximation (TPFA). There are several types of MPFA method for example MPFA O-method (Edwards and Rogers 1998; Aavatsmark et al. 1998; Aavatsmark 2002), MPFA L-method (Aavatsmark et al. 2008; Cao et al. 2009), MPFA U-method (Aavatsmark and Eigestad 2006), and MPFA Z-method (Nordbotten and Eigestad 2005). In this section, we only discuss the MPFA O-method. The convergence of MPFA has been examined theoretically (Klausen and Winther 2006; Agelas and Masson 2008) and numerically (Eigestad and Klausen 2005; Aavatsmark et al. 2006; 2007). MPFA method involves more point stencils than TPFA, e.g., a velocity is influenced by six neighboring cells for 2-D problem (Figure 1b). Therefore, the divergence of velocity involves 9-point stencil for 2-D problem (Figure 2). In this method, surface midpoints are introduced to ensure the linear variation and the continuity of pressure and flux. MPFA method shapes an interaction region (dashed lines) that is centered on each grid node and involving four adjacent cells for 2-D problem (Figure 3). The interaction region consists of four sub-cells, which connect the centers of the cells with the midpoints of the interfaces (edges) of the cells. For each sub-cell in an interaction region a linear pressure gradient is constructed through the cell-centered pressure and interface pressure. The velocity in TPFA is approximated by:

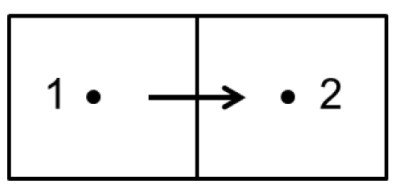

(a)

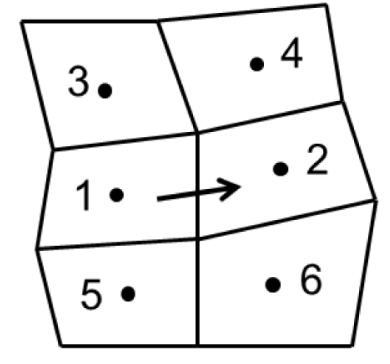

(b)

Figure 1-Two-point flux (a) and the multipoint flux (b) approximations. 


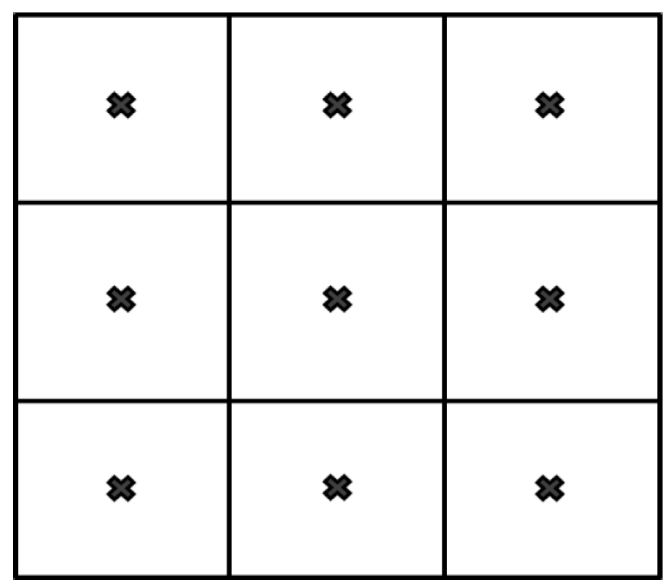

Figure 2-Cell stencil of a divergence of velocity in MPFA method.

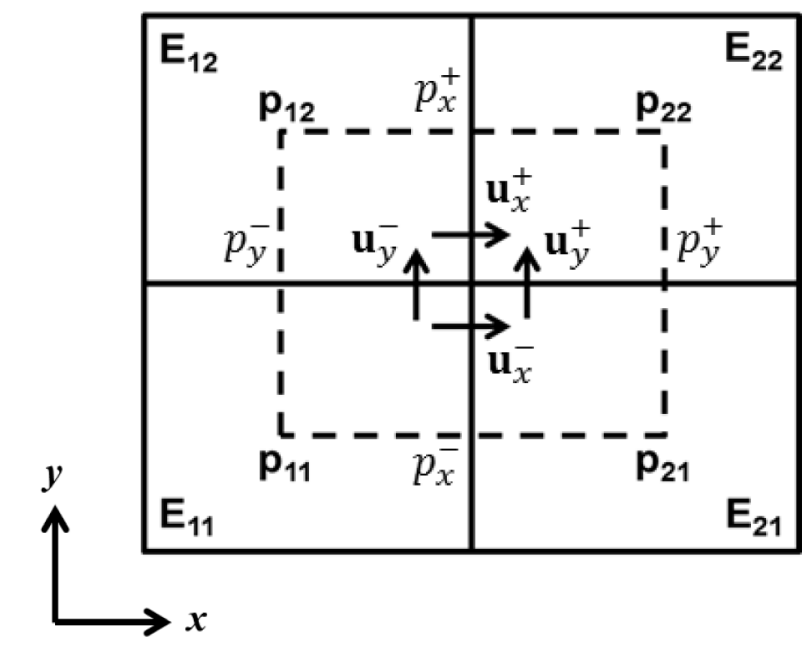

Figure 3-Interaction region in MPFA method for 2-D problem.

$$
u_{i} \approx T_{i j} \nabla p_{j}
$$

A velocity in MPFA is generally approximated by:

$$
u_{i} \approx \sum_{j \in \Omega} T_{i j} \nabla p_{j}
$$

here, Tij is transmissibility coefficients, $\nabla p j$ is pressure gradient, and $\Omega$ is the domain that depends on the dimensionality of the problem, e.g., a set of $\Omega$ comprises 6 cells for 2-D problem. In this work, MPFA method is applied to the advection terms of Eq. 26 and Eq. 27. To illustrate the method, let's consider the advection term of Eq. 27. Referring to Fig. 3, each velocity in each interaction region is calculated by:

$$
\begin{aligned}
& \boldsymbol{u}_{x}^{-}: u_{x}^{-}=-\alpha \frac{K_{f, 11}^{x x}}{\mu_{g, 11}} \frac{p_{x}^{-}-p_{f, 11}}{\frac{1}{2} \Delta x_{11}}-\alpha \frac{K_{f, 11}^{x y}}{\mu_{g, 11}} \frac{p_{y}^{-}-p_{f, 11}}{\frac{1}{2} \Delta y_{11}} \\
& u_{x}^{-}=-\alpha \frac{K_{f, 21}^{x x}}{\mu_{g, 21}} \frac{p_{f, 21}-p_{x}^{-}}{\frac{1}{2} \Delta x_{21}}-\alpha \frac{K_{f, 21}^{x y}}{\mu_{g, 21}} \frac{p_{y}^{+}-p_{f, 21}}{\frac{1}{2} \Delta y_{21}}
\end{aligned}
$$




$$
u_{x}^{+}
$$

$$
\begin{aligned}
& u_{x}^{+}=-\alpha \frac{K_{f, 12}^{x x}}{\mu_{g, 12}} \frac{p_{x}^{+}-p_{f, 12}}{\frac{1}{2} \Delta x_{12}}-\alpha \frac{K_{f, 12}^{x y}}{\mu_{g, 12}} \frac{p_{f, 12}-p_{y}^{-}}{\frac{1}{2} \Delta y_{12}} \\
& u_{x}^{+}=-\alpha \frac{K_{f, 22}^{x x}}{\mu_{g, 22}} \frac{p_{f, 22}-p_{x}^{+}}{\frac{1}{2} \Delta x_{22}}-\alpha \frac{K_{f, 22}^{x y}}{\mu_{g, 22}} \frac{p_{f, 22}-p_{y}^{+}}{\frac{1}{2} \Delta y_{22}}
\end{aligned}
$$

$u_{y}^{-}:$

$$
\begin{aligned}
& u_{y}^{-}=-\alpha \frac{K_{f, 11}^{y y}}{\mu_{g, 11}} \frac{p_{y}^{-}-p_{f, 11}}{\frac{1}{2} \Delta y_{11}}-\alpha \frac{K_{f, 11}^{y x}}{\mu_{g, 11}} \frac{p_{x}^{-}-p_{f, 11}}{\frac{1}{2} \Delta x_{11}} \\
& u_{y}^{-}=-\alpha \frac{K_{f, 12}^{y y}}{\mu_{g, 12}} \frac{p_{f, 12}-p_{y}^{-}}{\frac{1}{2} \Delta y_{12}}-\alpha \frac{K_{f, 12}^{y x}}{\mu_{g, 12}} \frac{p_{x}^{+}-p_{f, 12}}{\frac{1}{2} \Delta x_{12}}
\end{aligned}
$$

$u_{y}^{+}$

$$
\begin{aligned}
& u_{y}^{+}=-\alpha \frac{K_{f, 21}^{y y}}{\mu_{g, 21}} \frac{p_{y}^{+}-p_{f, 21}}{\frac{1}{2} \Delta y_{21}}-\alpha \frac{K_{f, 21}^{y x}}{\mu_{g, 21}} \frac{p_{f, 21}-p_{x}^{-}}{\frac{1}{2} \Delta x_{21}} \\
& u_{y}^{+}=-\alpha \frac{K_{f, 22}^{y y}}{\mu_{g, 22}} \frac{p_{f, 22}-p_{y}^{+}}{\frac{1}{2} \Delta y_{22}}-\alpha \frac{K_{f, 22}^{y x}}{\mu_{g, 22}} \frac{p_{f, 22}-p_{x}^{+}}{\frac{1}{2} \Delta x_{22}}
\end{aligned}
$$

where $\alpha=\gamma P f$ and $P_{f}$ in this $\alpha$ is known pressure from the previous time step, while $P f$ in the pressure gradient term is unknown. The other unknowns in Eq. 31 - Eq. 34 above are the interface pressures $\left(p_{x}^{+}, p_{x}^{-}, p_{y}^{+}, p_{y}^{-}\right)$and the velocities. From these equations, we eliminate the interface pressures so that the remaining unknowns are the cell-centered pressures and the velocities. In each interaction region, we solve the systems of linear equations as follows:

$$
\mathbf{B} \mathbf{u}=\mathbf{C} \mathbf{p}
$$

where $\mathbf{B}$ is expressed in the matrix form by

$$
\mathbf{B}=\frac{1}{2}\left[\begin{array}{ll}
B_{11} & B_{12} \\
B_{21} & B_{22}
\end{array}\right]
$$

where

$$
\begin{gathered}
B_{11}=\left[\begin{array}{cc}
\left(B_{11}^{x x} \Delta x_{1}+B_{21}^{x x} \Delta x_{2}\right) & 0 \\
0 & \left(B_{12}^{x x} \Delta x_{1}+B_{22}^{x x} \Delta x_{2}\right)
\end{array}\right] \\
B_{22}=\left[\begin{array}{cc}
\left(B_{11}^{y y} \Delta y_{1}+B_{12}^{y y} \Delta y_{2}\right) & 0 \\
0 & \left(B_{21}^{y y} \Delta y_{1}+B_{22}^{y y} \Delta y_{2}\right)
\end{array}\right] \\
B_{21}=\left[\begin{array}{ll}
B_{11}^{y x} \Delta y_{1} & B_{12}^{y x} \Delta y_{2} \\
B_{21}^{y x} \Delta y_{1} & B_{22}^{y x} \Delta y_{2}
\end{array}\right]
\end{gathered}
$$




$$
B_{12}=\left[\begin{array}{ll}
B_{11}^{x y} \Delta x_{1} & B_{21}^{x y} \Delta x_{2} \\
B_{12}^{x y} \Delta x_{1} & B_{22}^{x y} \Delta x_{2}
\end{array}\right]
$$

C is the matrix of coefficients,

$$
\mathbf{C}=\left[\begin{array}{cccc}
-1 & 1 & 0 & 0 \\
0 & 0 & -1 & 1 \\
-1 & 0 & 1 & 0 \\
0 & -1 & 0 & 1
\end{array}\right]
$$

$\mathbf{u}$ is the vector of unknowns velocities,

$$
\mathbf{u}=\left[\begin{array}{llll}
u_{x}^{-} & u_{x}^{+} & u_{y}^{-} & u_{y}^{+}
\end{array}\right]^{T}
$$

$\mathbf{p}$ is the vector of unknown pressures,

$$
\mathbf{p}=\left[\begin{array}{llll}
p_{11} & p_{21} & p_{12} & p_{22}
\end{array}\right]^{T}
$$

We obtain the solution for the fluxes in each node by solving Eq. 35. There are two velocities at each boundary of two neighboring cells. These two velocities are then averaged so that the velocity at the mid-point of the boundary is obtained. In other words, we return to the framework of TPFA. Assuming the domain consists of $m \times n$ cells then there are $(m+1) \times(n+1)$ interaction regions. This means that there are $4 \times(m+1) \times(n+1)$ velocities that must be calculated in all interaction regions.

\section{Experimenting Pressure Field Approach}

In this work, the governing equations, i.e., Eq. 26 and Eq. 27 presented in the shale gas modeling section are solved using the experimenting pressure field approach. The discretized, algebraic pressure equations are then written in the matrix form as a matrix of coefficients. Constructing the matrix of coefficients is not straightforward and errors can easily be made when it comes to coding because it involves large expressions of set of algebraic pressure equations. This becomes more challenging when the discretized form involves more point stencils as in the case of multipoint flux approximation (MPFA) method where it leads to 9-and 27-point stencil for 2-D and 3-D problems, respectively. The experimenting pressure field approach was introduced by Sun et al. (2012) to resolve that issue. This approach generates a set of predefined pressure fields in such a way that the undetermined coefficients are calculated from the experimenting pressure fields. The experimenting pressure field approach has been successfully implemented to solve incompressible single and two-phase flow problems (Sun et al. 2012; Negara 2015; Negara et al. 2013, 2014, 2015; Salama et al. 2013, 2014, In press). In this paper, we extend the application of experimenting pressure field approach to solve the compressible single-phase gas flow in shale reservoirs. In principle the implementation is very similar to the incompressible flow case. The only difference is the mass accumulation term in the compressible flow model. The mass accumulation term is an additional value to the values in the diagonal elements of the matrix of coefficients produced by the advection term. To illustrate the technique, we recall the pressure equations for the matrix and fracture (Eq. 26 and Eq. 27, respectively) and write them in the temporal discretization forms as follows:

$$
\begin{gathered}
A_{1} \frac{p_{m}^{t+1}-p_{m}^{t}}{\Delta t}+\nabla \cdot\left(-A_{2} \nabla p_{m}^{t+1}\right)=-A_{3} p_{m}^{t+1}+A_{3} p_{f}^{t+1} \\
A_{4} \frac{p_{f}^{t+1}-p_{f}^{t}}{\Delta t}+\nabla \cdot\left(-A_{\mathbf{5}} \nabla p_{f}^{t+1}\right)=A_{3} p_{m}^{t+1}-A_{3} p_{f}^{t+1}+\bar{q}
\end{gathered}
$$

where

$$
A_{1}=\phi_{m} \gamma+\frac{\left(1-\phi_{m}\right) M_{g} p_{L} V_{L} \rho_{s}}{V_{s t d}\left(p_{L}+p_{m}\right)^{2}}
$$




$$
\begin{gathered}
A_{2}=\gamma \frac{\mathbf{K}_{m}\left(p_{m}+b_{m}\right)}{\mu_{g}} \\
A_{3}=\frac{K_{m} \rho_{g} \sigma}{\mu_{g}} \\
A_{4}=\phi_{f} \gamma \\
A_{5}=\gamma p_{f} \frac{\mathbf{K}_{f}}{\mu_{g}}
\end{gathered}
$$

From Eq. 44 and Eq. 45, one obtains a global system of matrices as below:

$$
\left[\begin{array}{cc}
\left(\left(\frac{A_{1}}{\Delta t}+A_{3}\right) \mathbf{I}+\mathbf{A}_{2}\right) & -A_{3} \mathbf{I} \\
-A_{3} \mathbf{I} & \left(\left(\frac{A_{4}}{\Delta t}+A_{3}\right) \mathbf{I}+\mathbf{A}_{\mathbf{5}}\right)
\end{array}\right]\left[\begin{array}{c}
\mathbf{p}_{m}^{t+1} \\
\mathbf{p}_{f}^{t+1}
\end{array}\right]=\left[\begin{array}{c}
A_{1} \frac{\mathbf{p}_{m}^{t}}{\Delta t} \\
A_{4} \frac{\mathbf{p}_{f}^{t}}{\Delta t}+\overline{\mathbf{q}}
\end{array}\right]
$$

here, $\mathbf{I}$ is the identity matrix. Before solving the global system of matrices in Eq. 51, we localize the problem in order to construct the matrices of coefficients $\mathbf{A}_{2}$ and $\mathbf{A}_{5}$ by using the experimenting pressure field approach. Matrices $\mathbf{A}_{2}$ and $\mathbf{A}_{5}$ are constructed based on the advection term (divergence term) of Eq. 26 and Eq. 27, respectively. Using the same concept of implementation of experimenting pressure field approach to construct the matrix of coefficients in the incompressible flow model (Sun et al. 2012; Negara 2015; Negara et al. 2013, 2014, 2015; Salama et al. 2013, 2014, In press), the matrix of coefficients $\mathbf{A}_{5}$ is constructed as follows:

Consider the advection term of Eq. 27:

$$
\nabla \cdot \mathbf{u}=\nabla \cdot\left(-\gamma p_{f}\left(\frac{\mathbf{K}_{f}}{\mu_{g}} \nabla p_{f}\right)\right)=\bar{q}
$$

Assuming a domain of $\mathrm{m} \times \mathrm{n}$ cells, one obtains a set of algebraic equations as follows

$$
\sum_{j=1}^{m n} a_{i j} p_{f_{j}}=\bar{q}_{j} \pm c_{k} p_{f_{k}}^{B}
$$

where $a_{i j}$ is the coefficients that depend on the permeability, viscosity, grids and $\gamma$. The $p_{f, j}$ and $q_{j}$ are the unknown fracture pressures and sources or sinks in the $j^{\text {th }}$ cell over all $m \times n$ cells in the domain, respectively. Meanwhile, $c_{k} p_{f_{k}}^{B}$ contains information about the boundary conditions. Defining a vector function, $\mathbf{f}(\mathbf{p})$, which is constructed based on Eq. 53, one obtains

$$
\mathbf{f}(\mathbf{p})=\sum_{j=1}^{m n} a_{i j} p_{f_{j}} \pm \bar{q}_{j} \pm c_{k} p_{f_{k}}^{B}
$$

for $i=j=1,2, \ldots, m n$, and $k=1,2, \ldots, 2(m+n)$. In this approach, a set of experimenting pressure fields is given to Eq. 54. If the given experimenting pressure fields are correct then $\mathbf{f}(\mathbf{p})$ is a vector of zeros $(\mathbf{f}(\mathbf{p})=\mathbf{0})$. Else, there will be a residual equal to the difference between the divergence of the total flux and the sources or sinks,

$$
\mathbf{R}=\nabla \cdot \mathbf{u}-\bar{q}
$$

Thus, 


$$
\mathbf{f}(\mathbf{p})=\mathbf{R}
$$

Eq. 56 can be written as

$$
\sum_{j=1}^{m n} a_{i j} p_{f_{j}} \pm \bar{q}_{j} \pm c_{k} p_{f_{k}}^{B}=R_{i}
$$

Referring to Eq. 55, one obtains

$$
\sum_{j=1}^{m n} a_{i j} p_{f_{j}} \pm c_{k} p_{f_{k}}^{B}=(\nabla \cdot \mathbf{u})_{i}
$$

In this technique, we use Eq. 58 to populate the domain with the experimenting pressure fields in every two cells so that the values will be one in the cell of interest and zeros elsewhere. For 2-D problem, in the case of TPFA with 5-point stencil, the $i^{\text {th }}$ experimenting pressure field affects only four neighboring cells, while it affects eight neighboring cells for MPFA with 9-point stencil. Rewriting Eq. 58 as follows

$$
\sum_{j=1}^{m n} a_{i j} p_{f_{j}}=(\nabla \cdot \mathbf{u})_{i} \pm c_{k} p_{f_{k}}^{B}
$$

and letting

$$
\mathbf{p}_{f}^{k}=\left[\begin{array}{llllll}
0 & 0 & \ldots & 1 & \ldots & 0
\end{array}\right]_{m n}^{T}, \quad k=1,2, \ldots, m n
$$

where $\mathbf{p}_{f}^{k}$ is the $k$-fracture pressure field that is one at cell- $k$ and zeros elsewhere. Thus, the coefficients in the matrix $\mathbf{A}_{5}$ in Eq. 51 are:

$$
a_{i j}=(\nabla \cdot \mathbf{u})_{i}^{\mathbf{p}_{f}^{k}} \pm c_{k} p_{f_{k}}^{B}
$$

Similarly, we apply the experimenting pressure field approach to construct the matrix of coefficients $\mathbf{A}_{2}$ in Eq. 51. The application of this technique in constructing the matrix of coefficients that involves many point stencils, as in the case of MPFA, and in a large system of matrices as shown in Eq. 51 is greatly beneficial. It minimizes mistakes in the coding because the matrix of coefficient is constructed automatically within the solver. This makes the code more robust.

\section{Numerical Results and Discussions}

In this section, we present a numerical experiment of shale gas modeling with different anisotropy orientations. We consider a $61 \mathrm{~m} \times 41 \mathrm{~m}$ domain, which is discretized into $61 \times 41$ non-uniform meshes. The mesh size is reduced as it gets closer to the well. A single production well is located in the middle of the domain as shown in Fig. 4. The gas is produced from the fracture system because it has higher permeability than the matrix where the gas flows more easily. The bottom of the well is located in the fracture system. No-flow boundary conditions are imposed on all sides. The simulation time for the numerical experiment is 3 years. Basic assumptions are used in the model. These assumptions are: single phase (gas only), gas adsorption-desorption obeys Langmuir's model, no gravitational acceleration, rock is incompressible and has anisotropy property, no rock deformation (porosity remains the same) and temperature is constant. The model parameters including the reservoir and the gas properties are presented in Table 2. The elements of the permeability tensor are generated by the following formula, 


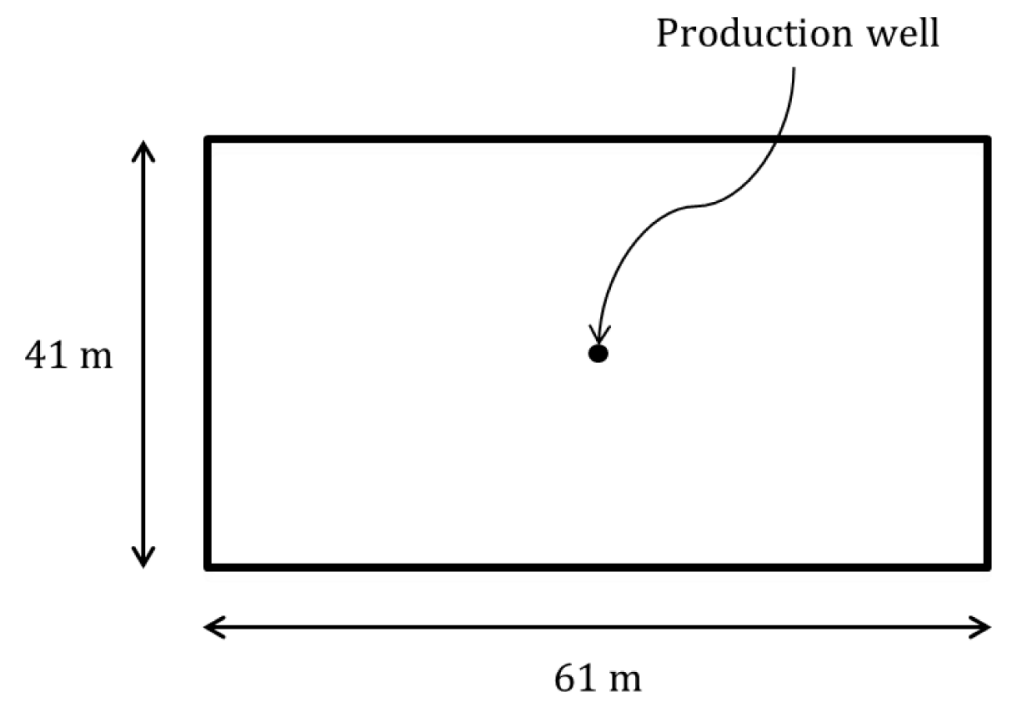

Figure 4-Setup of numerical experiment with single production well.

Table 2-Model parameters for the numerical experiment.

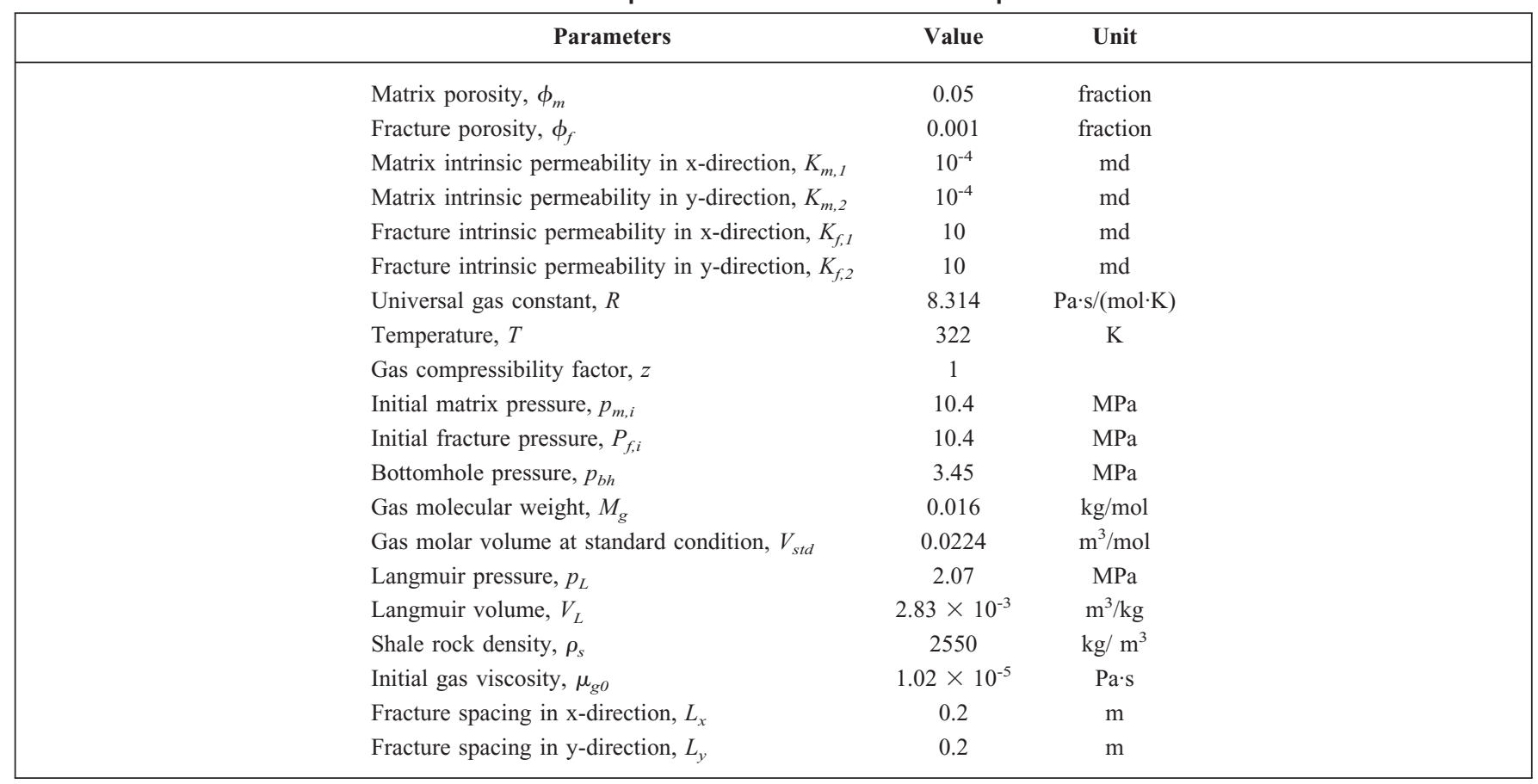

$$
\mathbf{K}=\left[\begin{array}{cc}
\cos \theta & -\sin \theta \\
\sin \theta & \cos \theta
\end{array}\right]\left[\begin{array}{cc}
K_{1} & 0 \\
0 & K_{2}
\end{array}\right]\left[\begin{array}{cc}
\cos \theta & \sin \theta \\
-\sin \theta & \cos \theta
\end{array}\right]
$$

where $K_{1}$ and $K_{2}$ are the permeabilities in the principal directions, horizontal and vertical, respectively and $\theta$ is the rotation angle of anisotropy. The value of $K_{1}$ is tipically greater than $K_{2}$ because the deposition of sediments induces the permeability to be dominant in one direction (i.e., horizontal direction) over other directions. The permeability tensor formulation is equivalent to the one in Fanchi (2008). Assuming anisotropy exists in both matrix and fracture systems and applying MPFA method on both equations to handle the full permeability tensor. We solve Eq. 26 and Eq. 27 using semi-implicit scheme. Fig. 5 and Fig. 7 show the pressure fields for the anisotropic and isotropic cases with different anisotropy orientations, $\theta=0^{\circ}, \theta=30^{\circ}, \theta=45^{\circ}, \theta=60^{\circ}$, and $\theta=90^{\circ}$, after 3 years of the production. These figures show 
that anisotropy plays an important role in dictating the pressure fields of both matrix and fracture systems. The initial pressure in both the matrix and the fracture is $10.4 \mathrm{MPa}$. After the well is opened, the pressure of the fracture declines rapidly, reaching a value close to the bottomhole pressure. As the well starts to produce gas, the pressure in the matrix declines from initial pressure at the outer boundary of the domain towards the bottomhole pressure at the center. The pressure gradient increases as we get closer to the well. The pressures in the matrix and in the fracture including boundaries continue to decline with time until they reach values close to the bottomhole pressure, 3.45 $\mathrm{MPa}$. When the pressures in the matrix and the fracture reach these values, no more gas can be produced from the reservoir. Figs. 9a-f depict the changes of pressures in matrix and fracture for $\theta=45$ at early-times (from $t_{1}$ to $t_{6}$ where $t_{1}<t_{2}<t_{3}<t_{4}<t_{5}$ $\left.<t_{\sigma}\right)$. The other anisotropic cases $\left(\theta=0^{\circ}, \theta=30^{\circ}, \theta=60^{\circ}\right.$, and $\left.\theta=90^{\circ}\right)$ as well as the isotropic case exhibit the same evolution pattern. At the end of three years of production, the pressure fields for all values of $\theta$ decline close to the bottomhole pressure. This is shown in Fig. 5 and Fig. 7. The $\theta=30^{\circ}, \theta=45^{\circ}$ and $\theta=60^{\circ}$ cases reach slightly lower pressures compared to $\theta=0^{\circ}, \theta=90^{\circ}$ and isotropic cases. The velocity distributions in the matrix and in the fracture are shown in Fig. 6 and Fig. 8, respectively. The gas production rate shown in Fig. 10 indicates that the pressures in the matrix and fracture decrease steeply during the first 200 days. Fig. 11 shows gas flow rate at early times and Fig. 12 shows the gas flow rate at late times. From these three figures we can see that most of the gas is produced in the first seven months. During this period of time, most of the produced gas is coming from the fracture network and very little is coming from the nanodarcy matrix. This implies that the effects of Knudsen diffusion and slip flow (Klinkenberg effect) are not significant in early period. After that, Knudsen diffusion and slip flow become more significant because they govern the gas flow in the matrix system. Fig. 11 shows that during the early-time production especially for the first 30 days, the anisotropic cases with $\theta=30^{\circ}, \theta=45^{\circ}$, and $\theta=60^{\circ}$ produce the highest gas flow rate compared to other anisotropic cases. From this figure, we can also see that the isotropic case produces the lowest gas flow rate compared to the anisotropic cases. After 200 days and to the end of production, however, the gas flow rate of those three anisotropic cases $(\theta=$ $30^{\circ}, \theta=45^{\circ}$, and $\theta=60^{\circ}$ ) become the lowest compared to the cases of $\theta=0^{\circ}$ and $\theta=90^{\circ}$ (see Fig. 10 and Fig. 12). It can also be seen that the gas flow rate of isotropic case becomes the highest compared to the anisotropic cases. With no-flow boundary conditions for all cases (i.e. total amount of gas in the reservoir is equal for all cases) and the gas flow rate being high at early time production, the gas flow rate will be lower at the late time production. In summary, for this example, we have seen that anisotropic cases yields higher gas production compared to the isotropic case as shown in Fig. 13. Fig. 14 and Fig. 15 show the cumulative production at mid and late time production. 


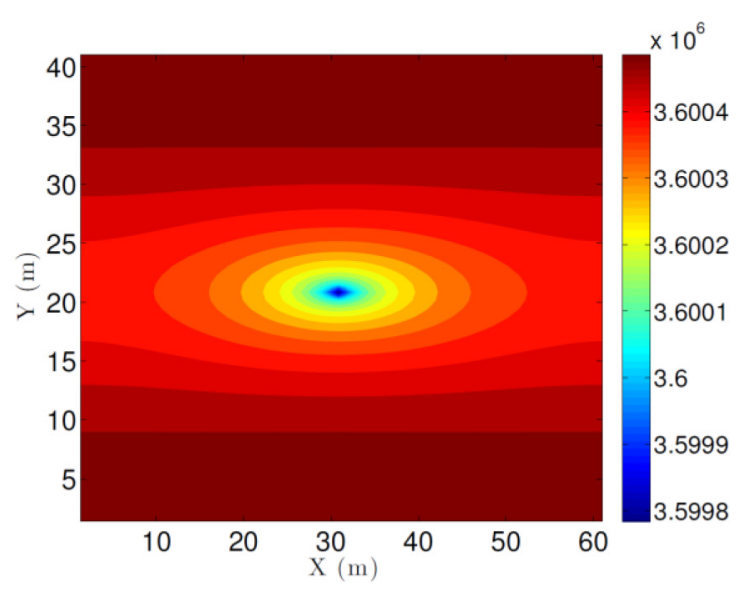

(a) $\theta=0^{\circ}$

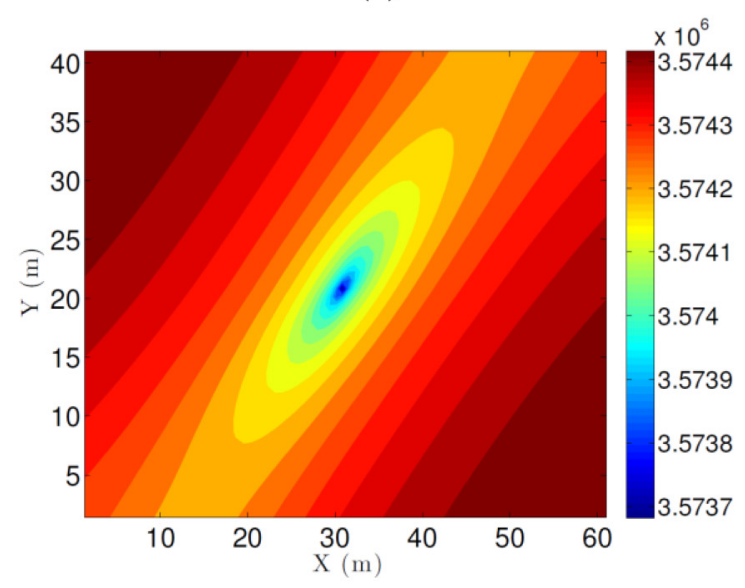

(c) $\theta=45^{\circ}$

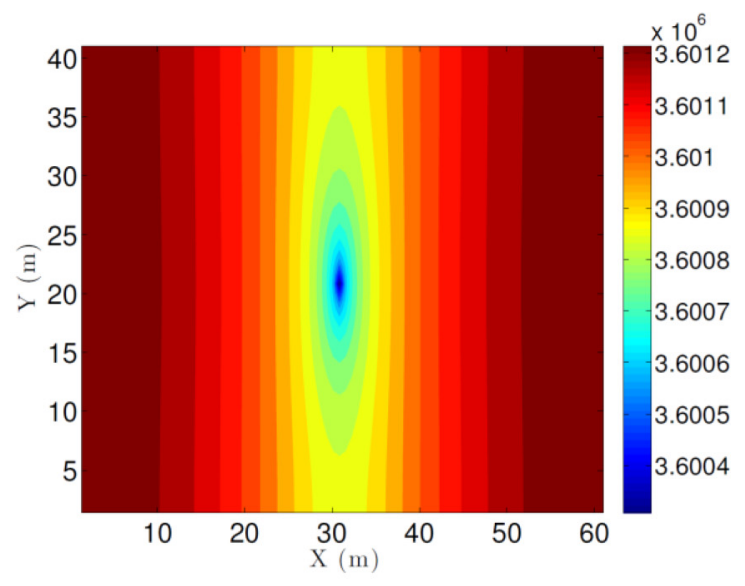

(e) $\theta=90^{\circ}$

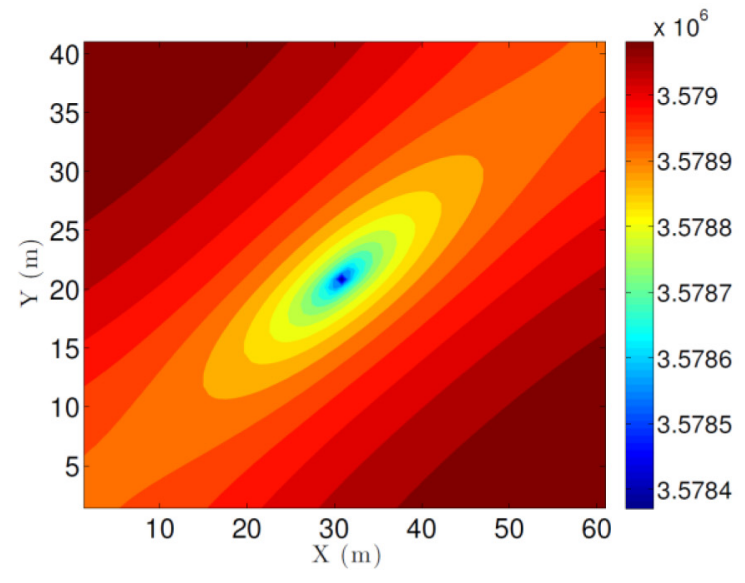

(b) $\theta=30^{\circ}$

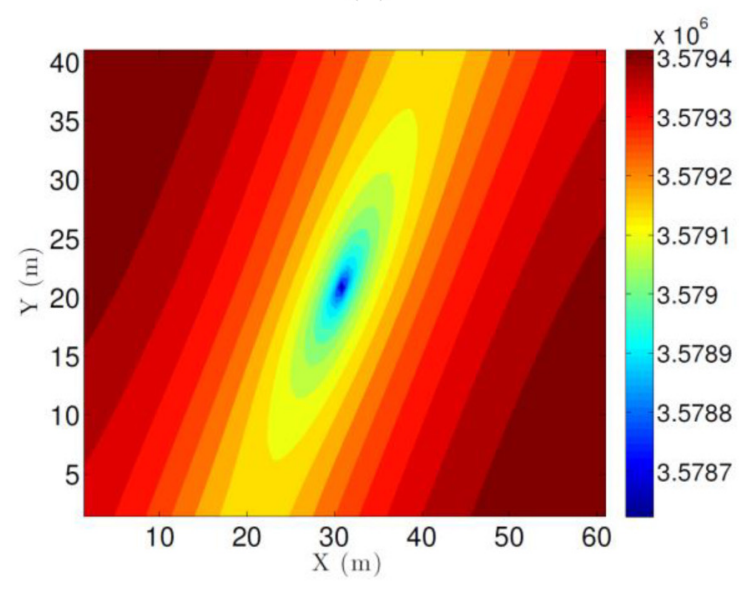

(d) $\theta=60^{\circ}$

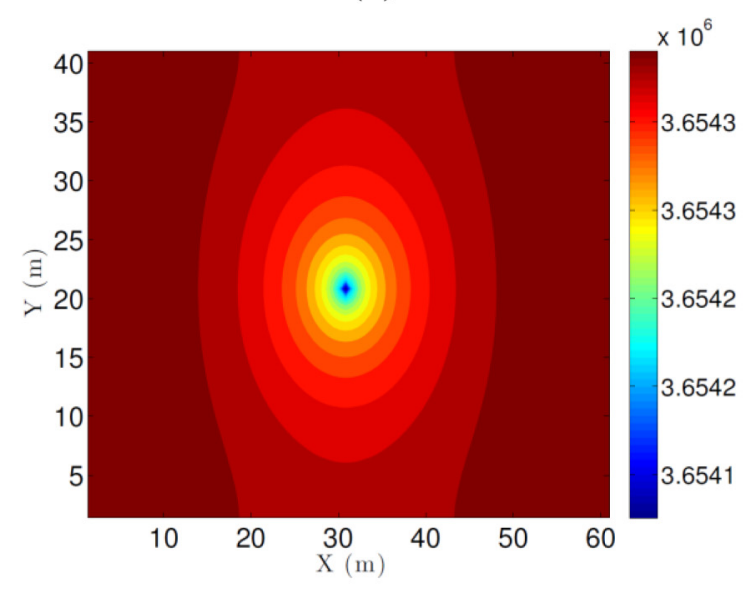

(f) isotropic

Figure 5-Pressure in the matrix after three years of production. 


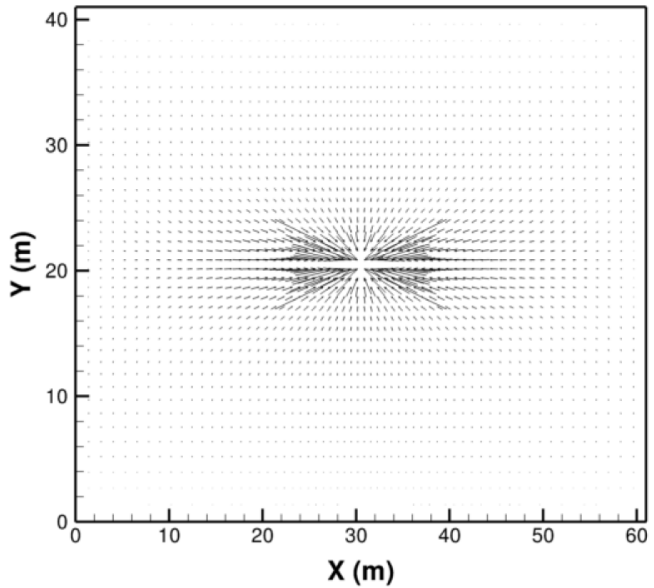

(a) $\theta=0^{\circ}$

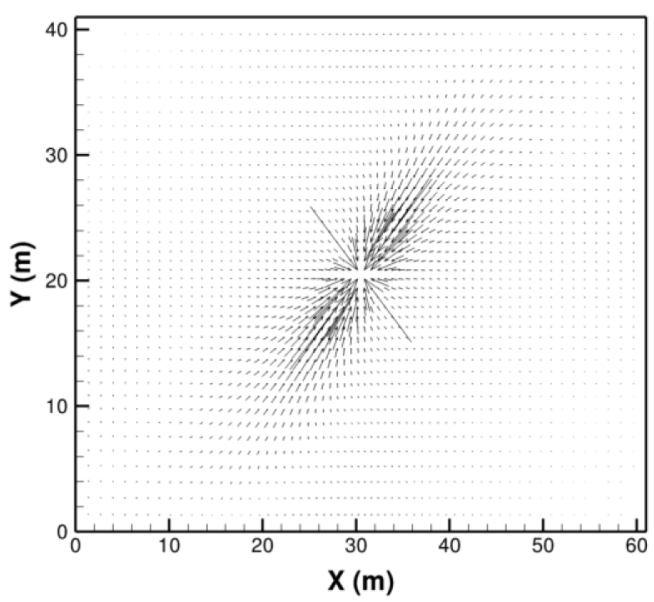

(c) $\theta=45^{\circ}$

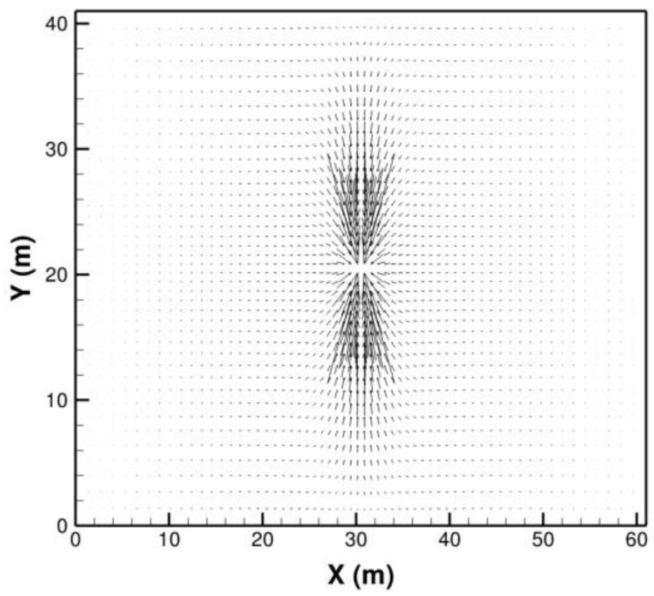

(e) $\theta=0^{\circ}$

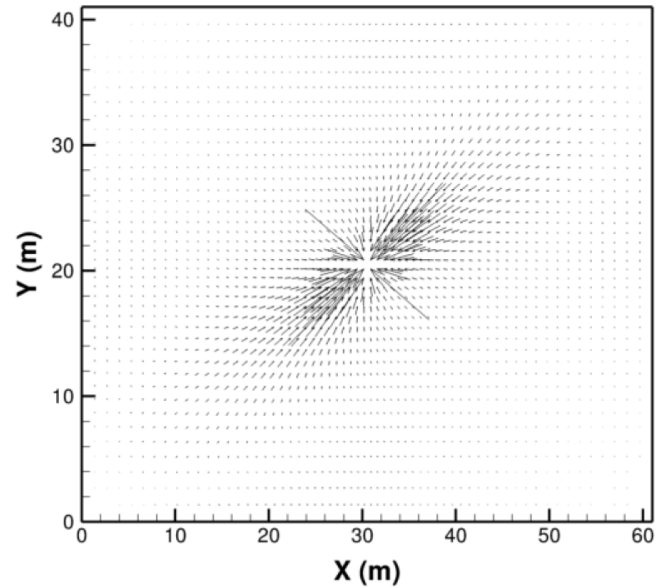

(b) $\theta=30^{\circ}$

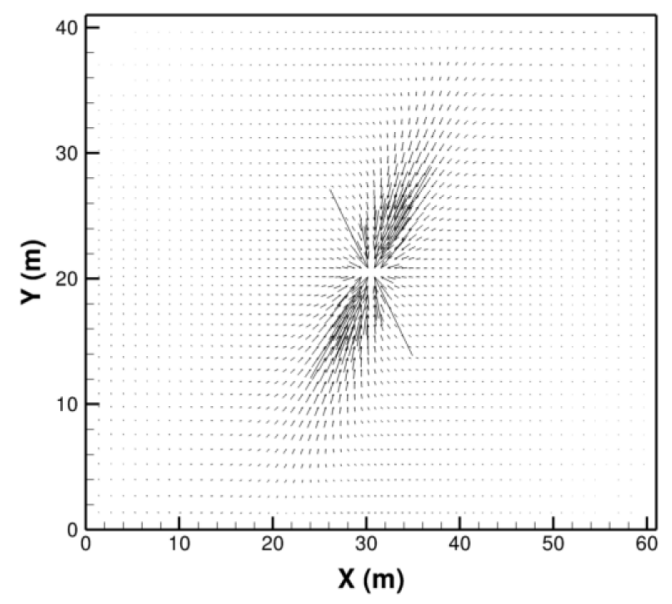

(d) $\theta=60^{\circ}$

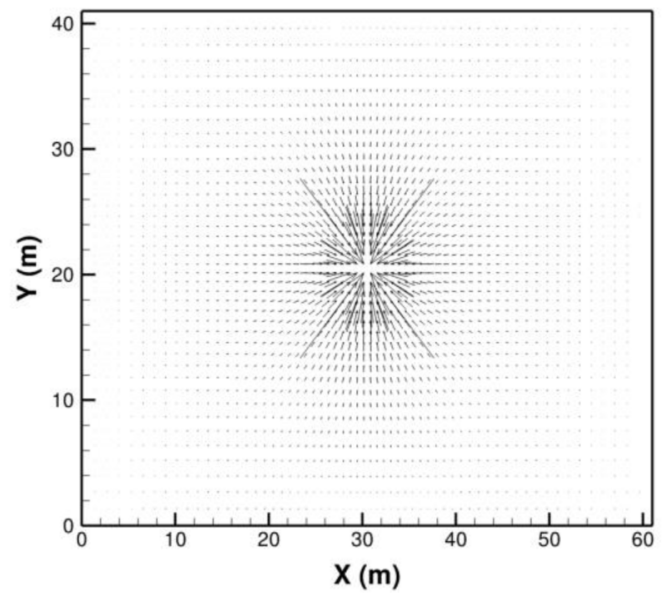

(f) isotropic

Figure 6-Velocity fields in the matrix after three years of production. 


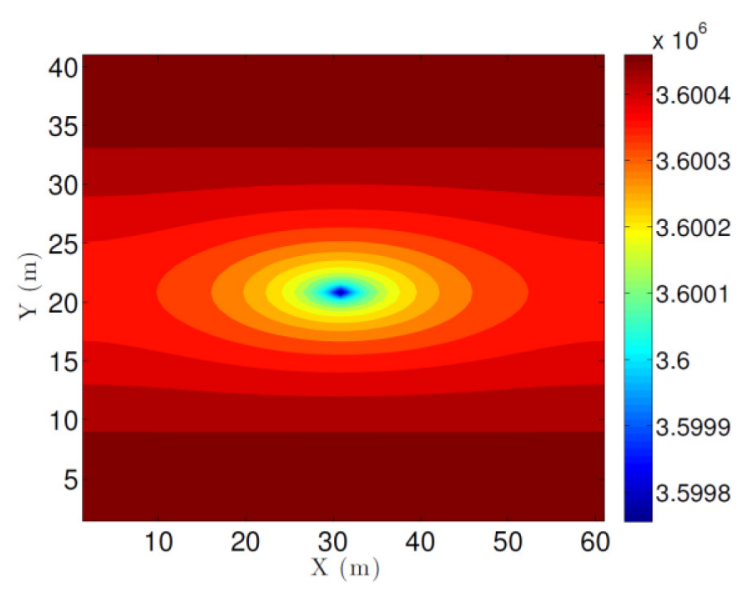

(a) $\theta=0^{\circ}$

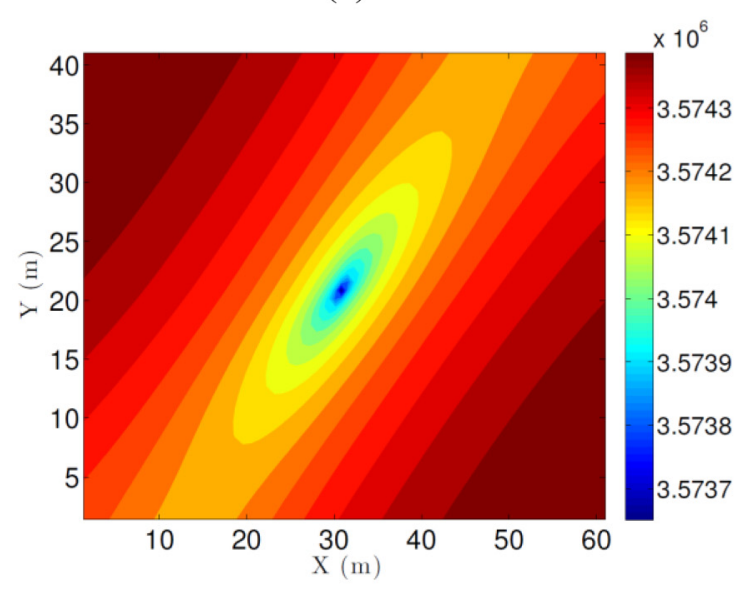

(c) $\theta=45^{\circ}$

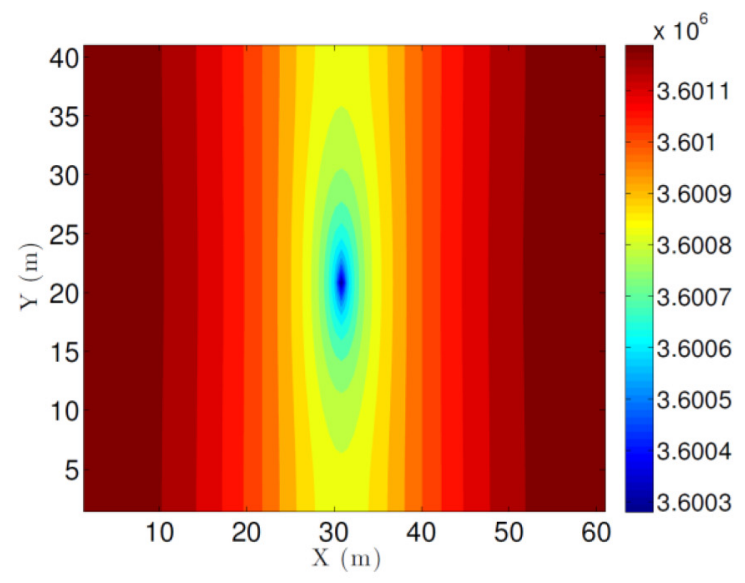

(e) $\theta=90^{\circ}$

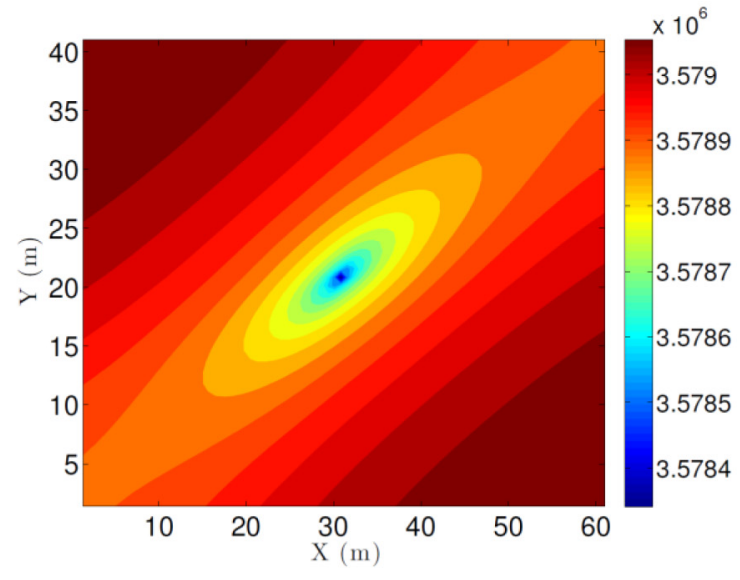

(b) $\theta=30^{\circ}$

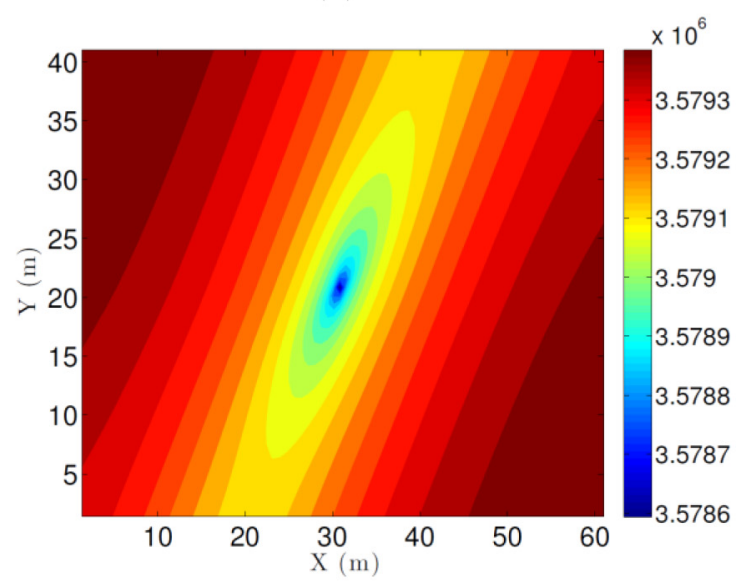

(d) $\theta=60^{\circ}$

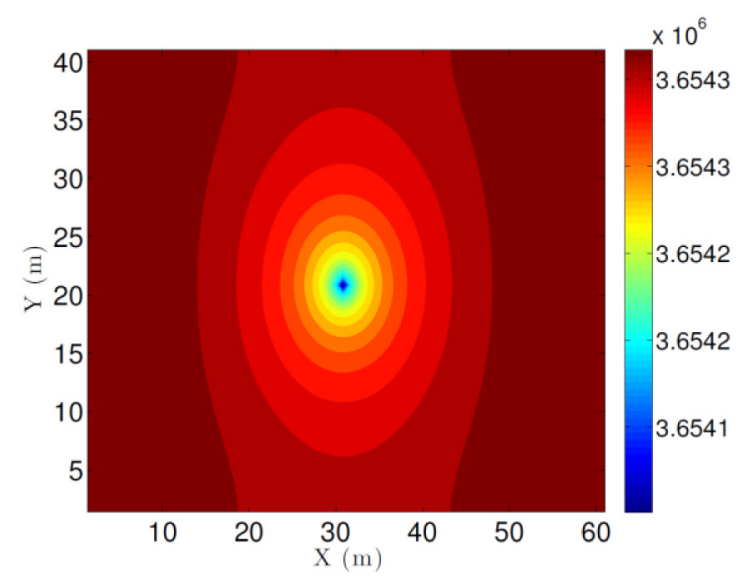

(f) isotropic

Figure 7-Pressure in the fracture after three years of production. 


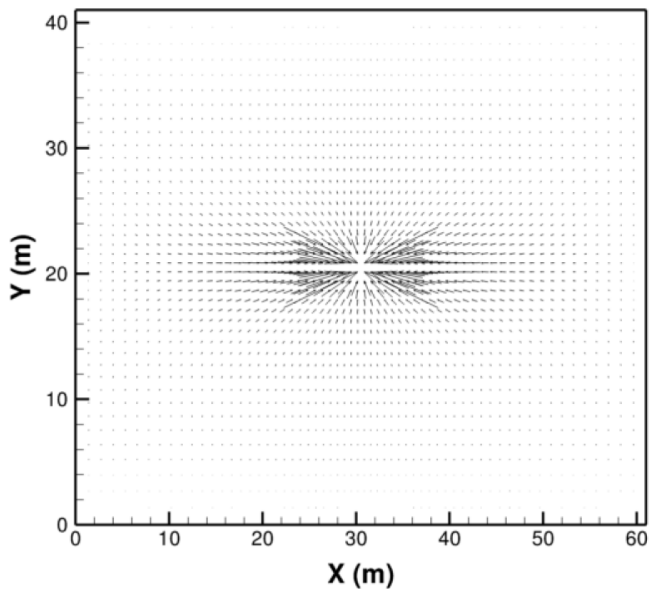

(a) $\theta=0^{\circ}$

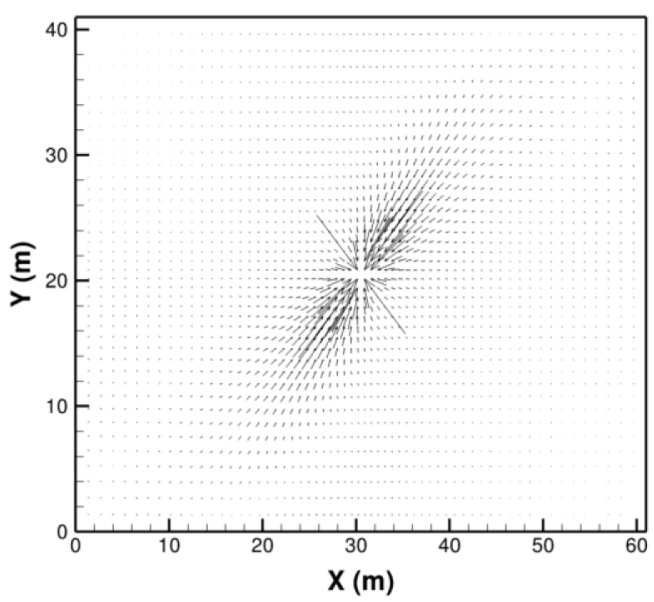

(c) $\theta=45^{\circ}$

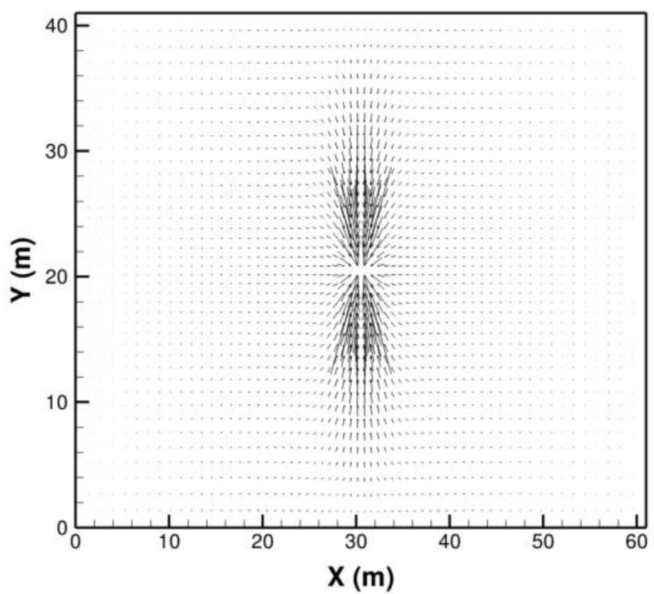

(e) $\theta=90^{\circ}$

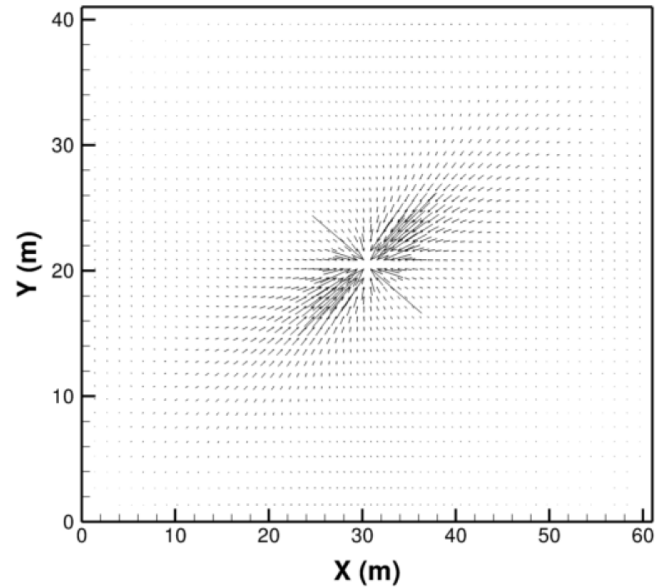

(b) $\theta=30^{\circ}$

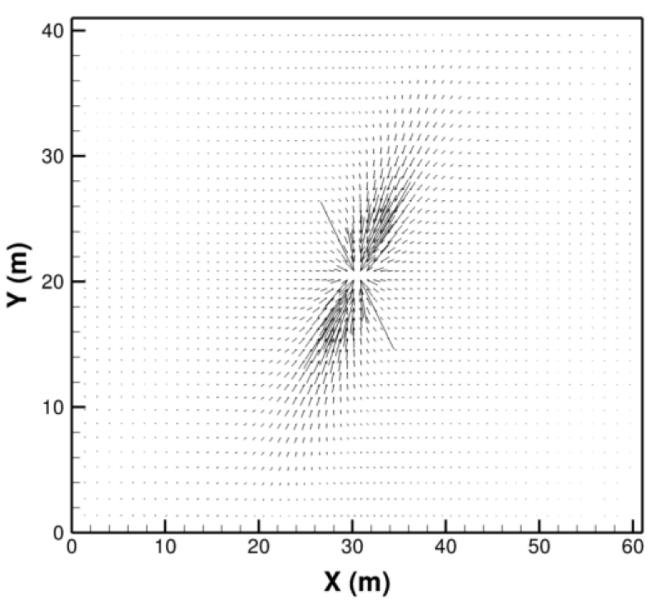

(d) $\theta=60^{\circ}$

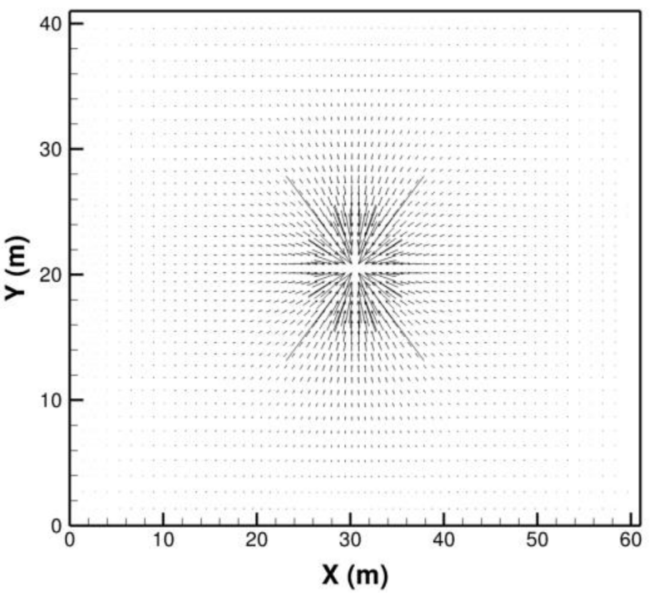

(f) isotropic

Figure $8-$ Velocity fields in the fracture after three years of production. 


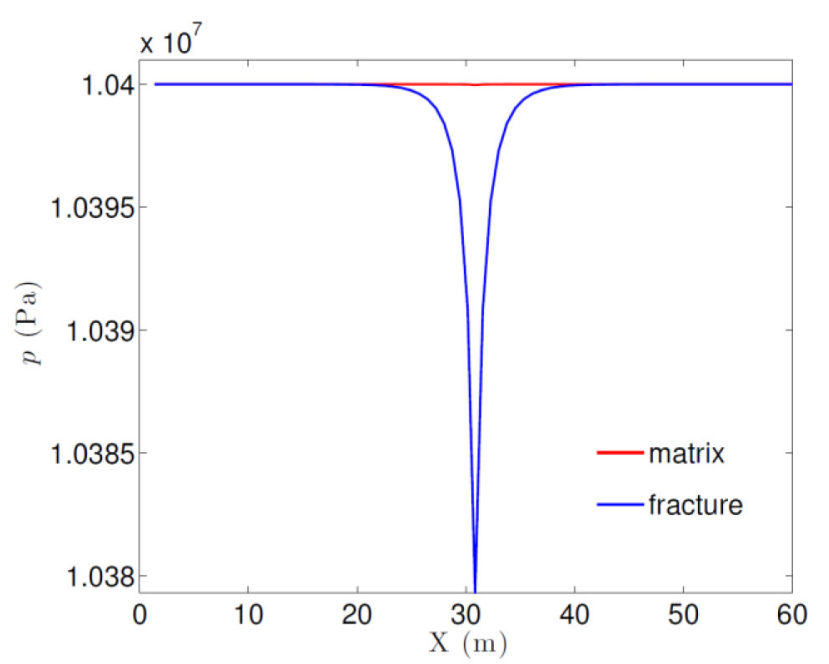

(a) $t_{1}$

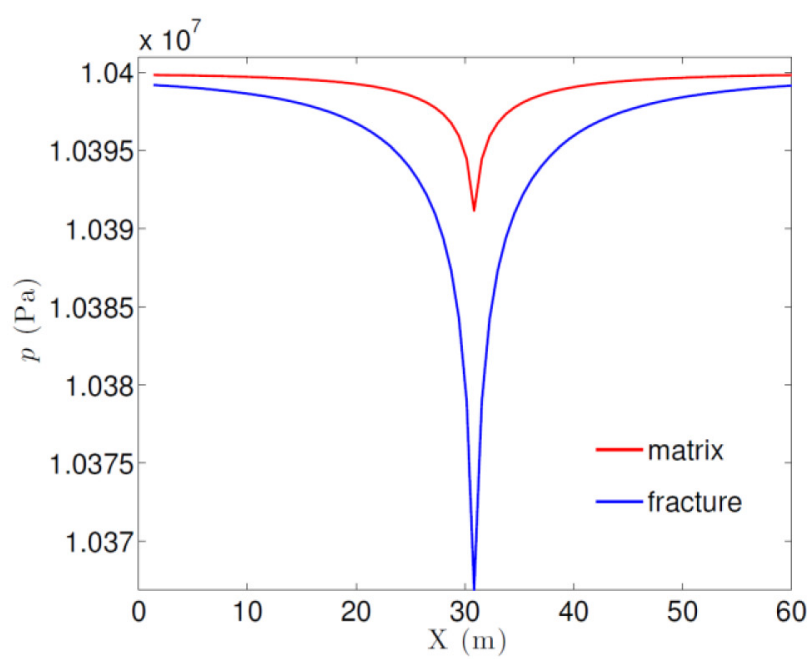

(c) $t_{3}$

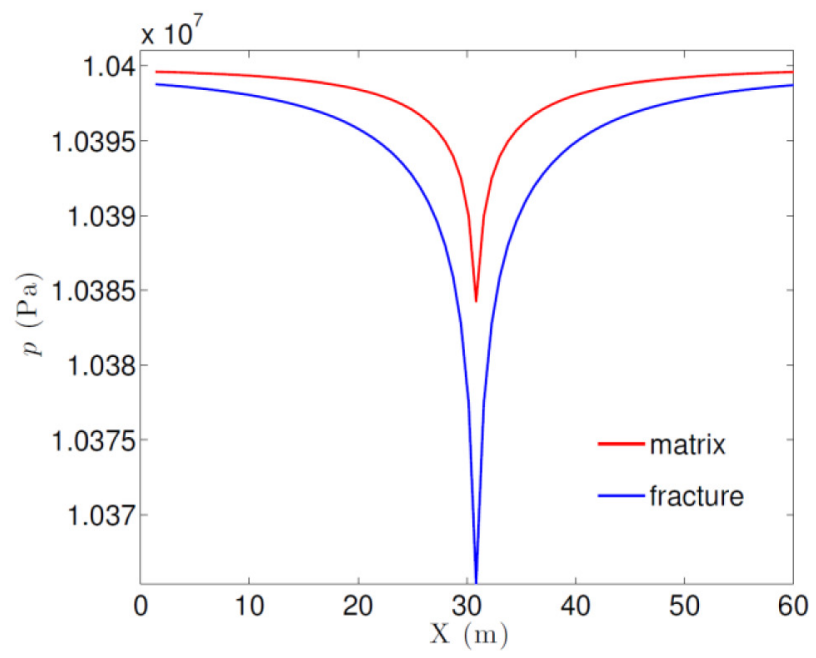

(e) $t_{5}$

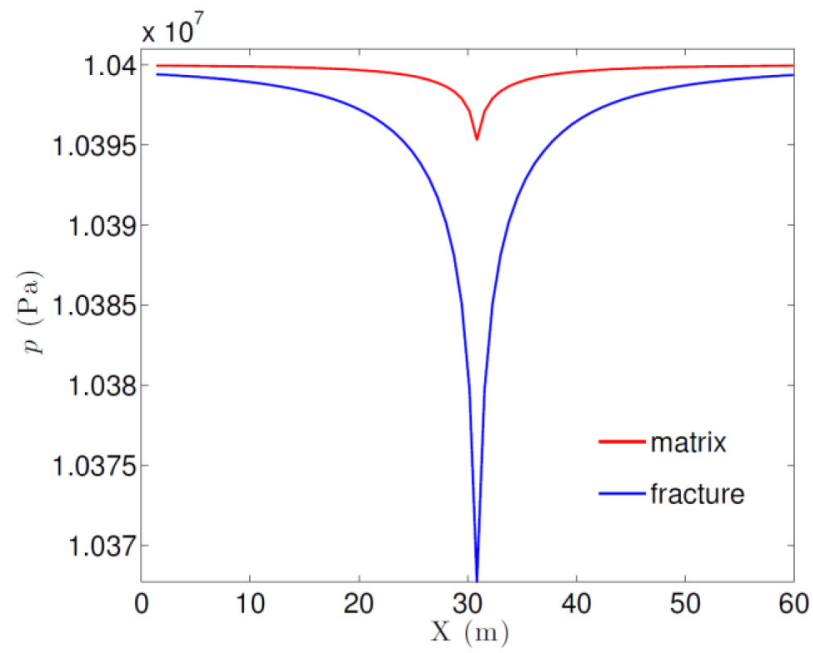

(b) $t_{2}$

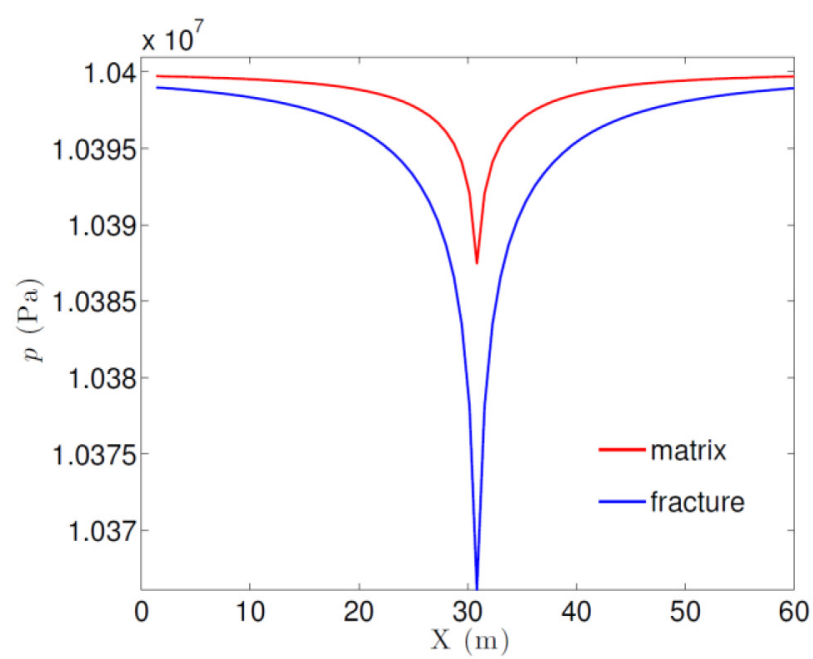

(d) $t_{4}$

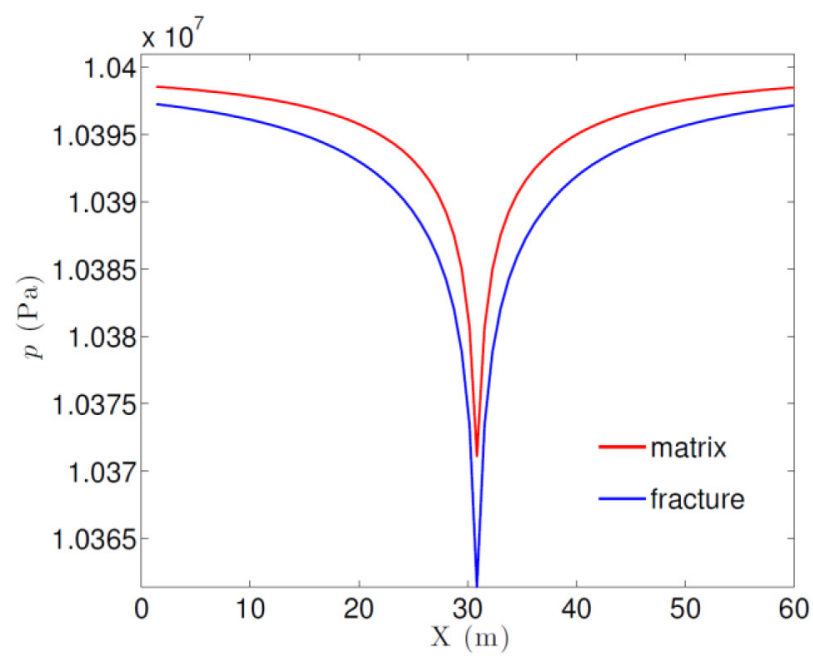

(f) $t_{6}$

Figure 9-Changes of pressures in the matrix and fracture for $\theta=45^{\circ}$ at early-time $\left(t_{1}<t_{2}<t_{3}<t_{4}<t_{5}<t_{6}\right)$. 


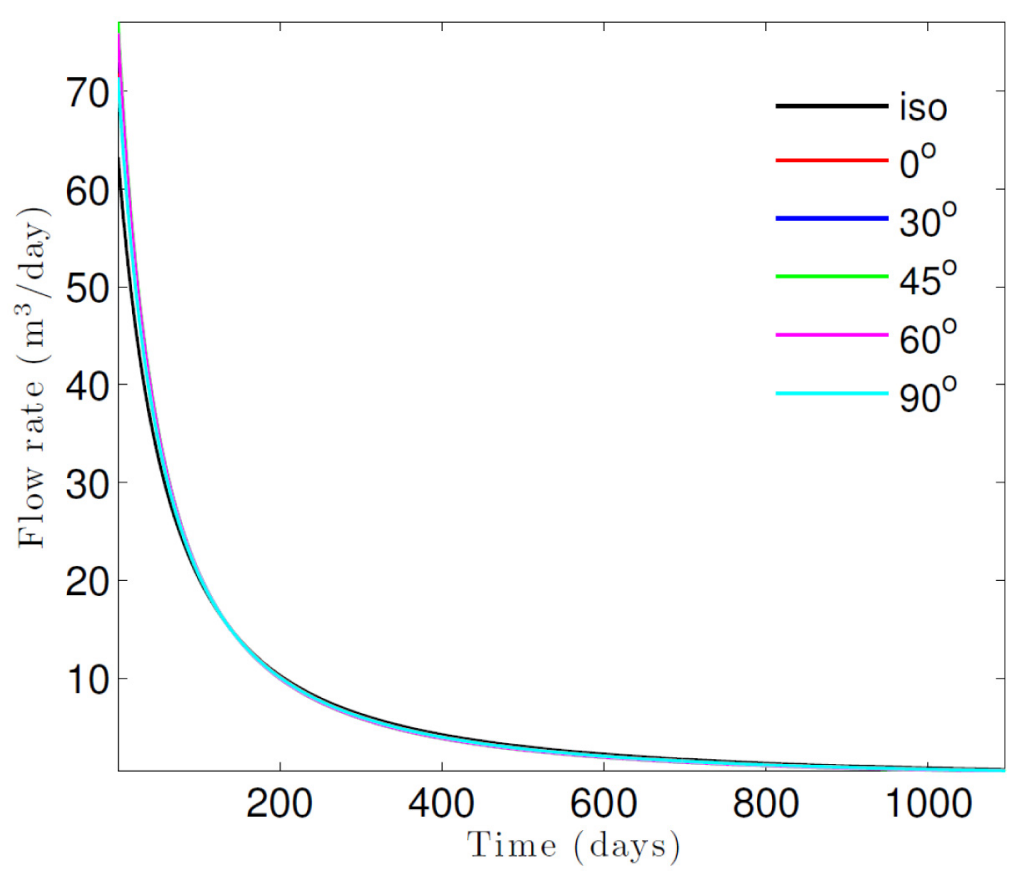

Figure $10-$ Gas flow rate versus time during three years of production.

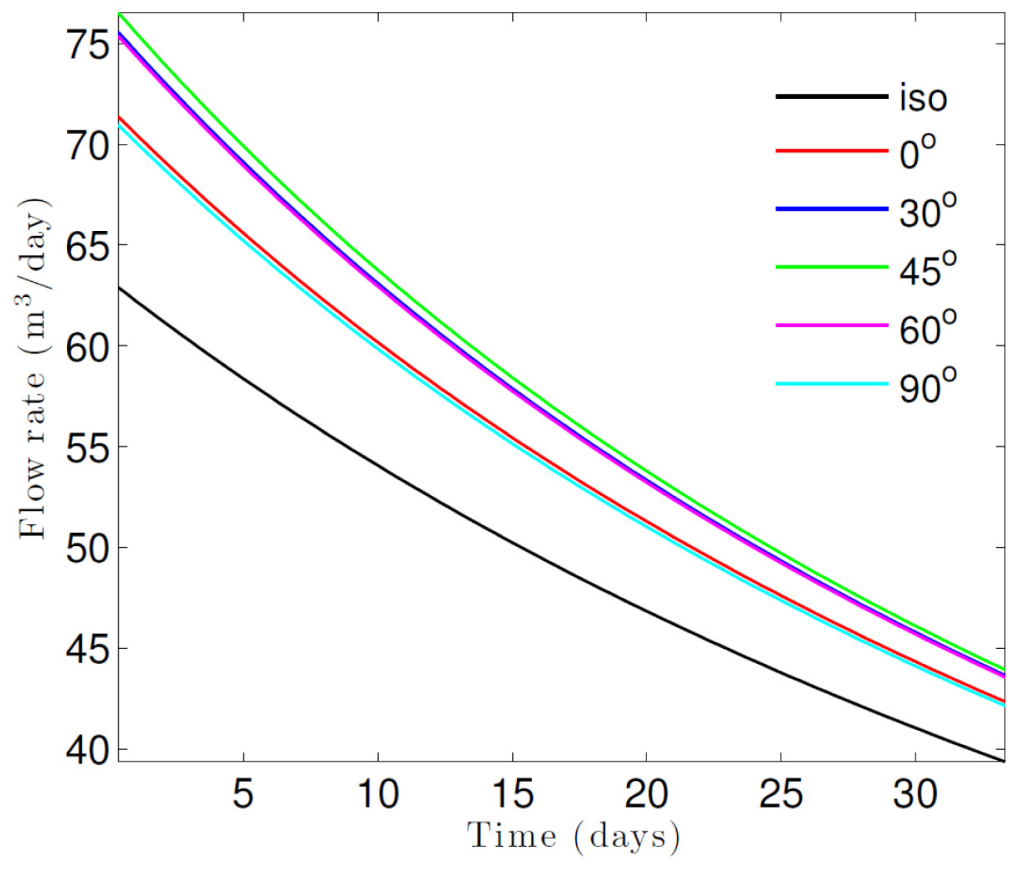

Figure $11-$ Gas flow rate (early time). 


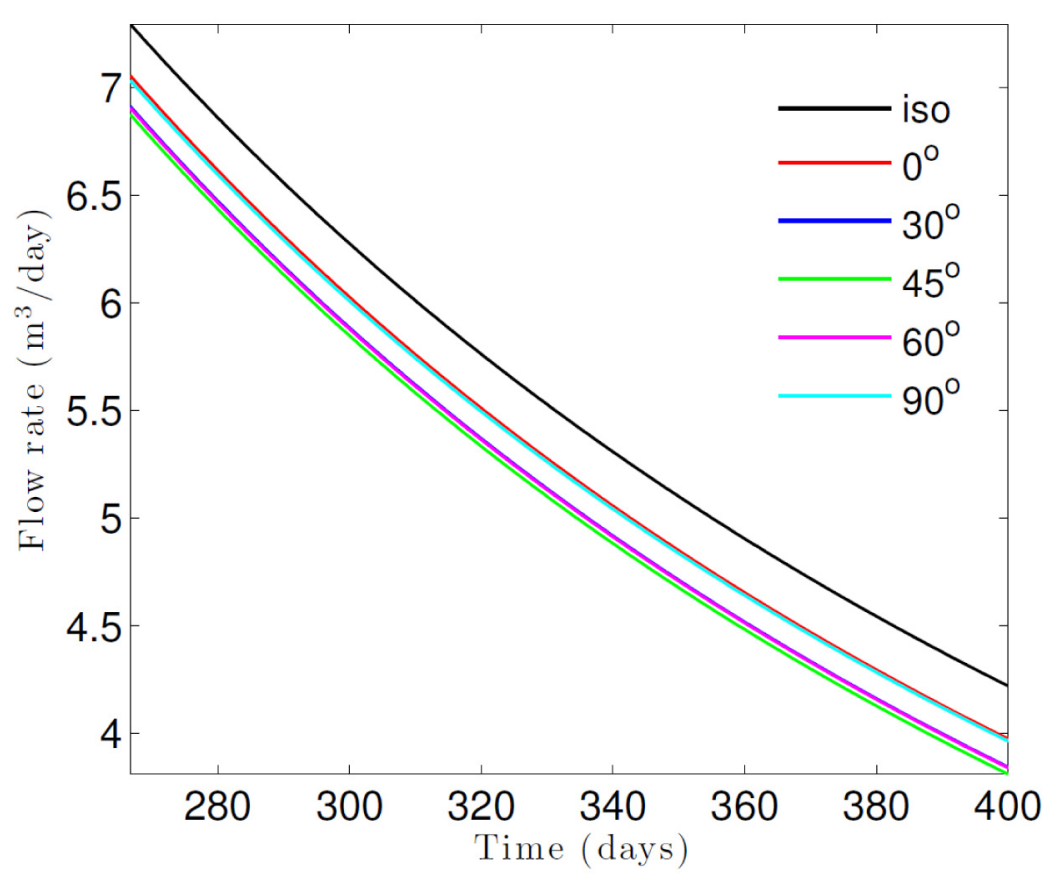

Figure 12-Gas flow rate (late time).

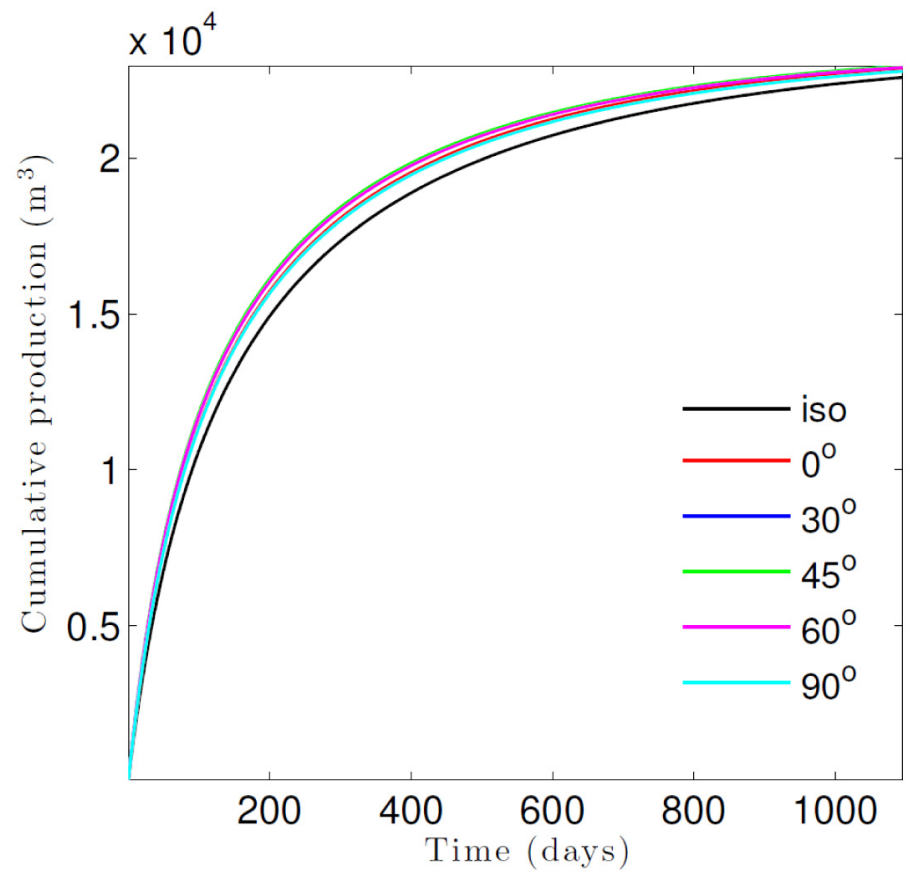

Figure 13-Cumulative gas production versus time during three years of production. 


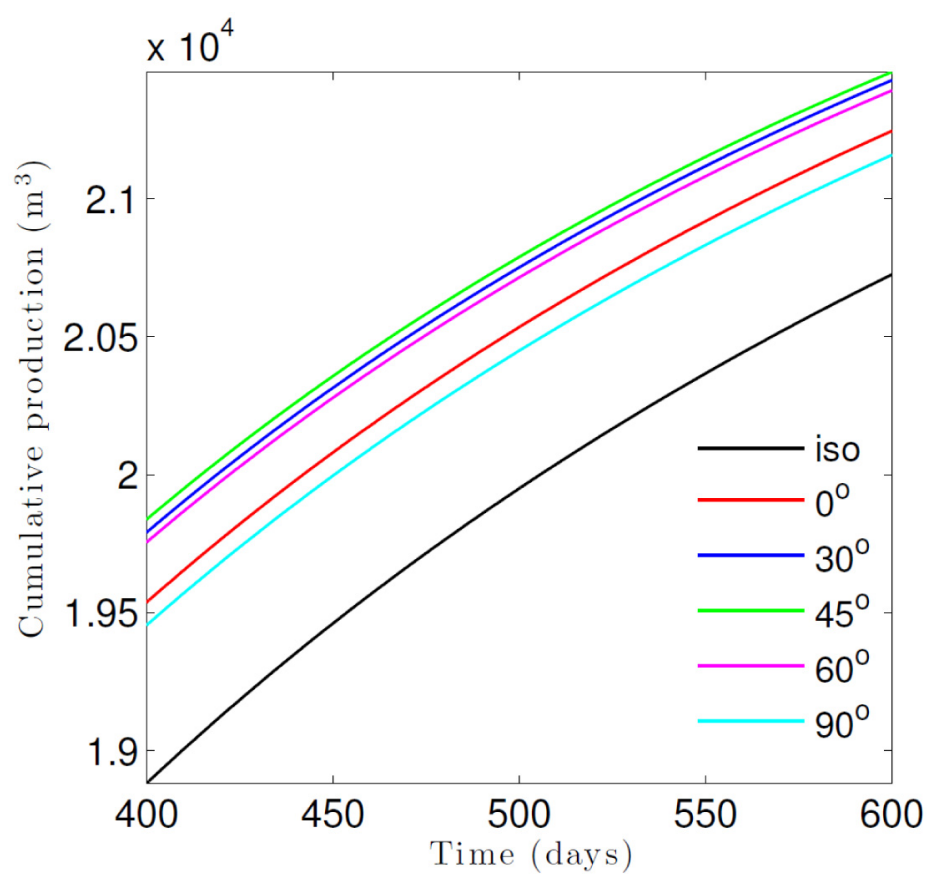

Figure 14-Cumulative gas production (mid time).

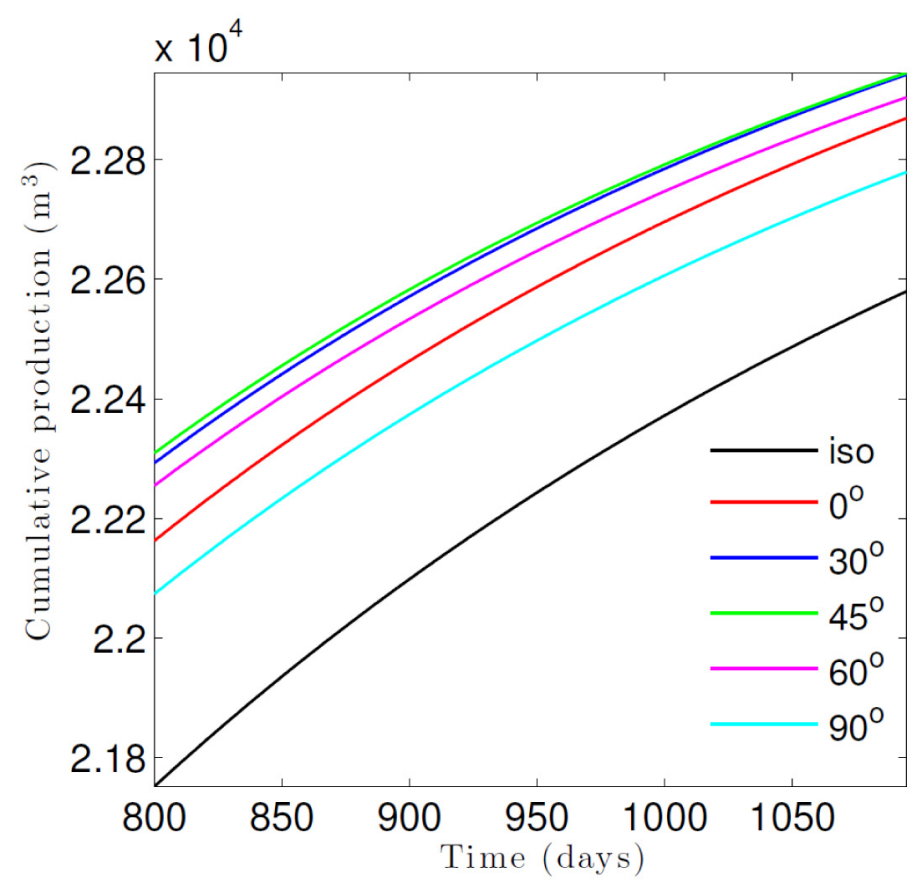

Figure 15-Cumulative gas production (late time).

\section{Conclusions}

A numerical investigation of natural gas flow in anisotropic porous media using the combined multipoint flux approximation (MPFA) and experimenting pressure field approach has been conducted. The MPFA method was used for handle the anisotropic shale reservoirs in order to handle the full apparent permeability tensor in the model. The experimenting pressure field approach was used to generate the matrix of coefficients automatically within the solver. The combined MPFA method with the experimenting pressure field approach was greatly beneficial to tackle the challenge in constructing the matrix 
of coefficients when the discretized form involves more point stencils as in the MPFA method, which leads to 9-and 27-point stencil for 2-D and 3-D problems, respectively. It is very important to include the anisotropy of the formation. Anisotropy along with the pressure gradient dictates the magnitude and direction of flow. A number of scenarios with different anisotropy orientations were run using the model to compare their results with the isotropic result. Both matrix and fracture systems were assumed anisotropic. The results show that anisotropy plays a crucial role in dictating the pressure fields as well as the gas flow streamlines. Furthermore, the numerical results emphasize the effects of anisotropy on the production rate and cumulative production. Incorporating anisotropy together with the gas flow mechanisms that occur in shale formations into the reservoir model is essential particularly for the purpose of maximizing gas production from shale reservoirs.

\section{Nomenclature}

$$
\begin{aligned}
& \mathrm{b}=\text { slip coefficient, } \mathrm{Pa} \\
& \mathrm{d}=\text { collision diameter of the gas molecule, } \mathrm{m} \\
& \mathrm{D}=\text { diameter of the pore, } \mathrm{m} \\
& \mathrm{D}_{\mathrm{k}} \quad=\text { Knudsen diffusion coefficient, } \mathrm{m}^{2} / \mathrm{s} \\
& \mathrm{g}=\text { gravitational acceleration, } \mathrm{m} / \mathrm{s} \\
& \mathrm{J}_{\mathrm{k}} \quad=\text { mass flux of component } i, \mathrm{~kg} / \mathrm{m}^{2} / \mathrm{s} \\
& \mathrm{K}_{\text {app }} \quad=\text { apparent permeability tensor, } \mathrm{md} \\
& \mathrm{K}_{\infty} \quad=\text { intrinsic permeability tensor, md } \\
& \mathrm{K}_{\mathrm{m}} \quad=\text { matrix intrinsic permeability tensor, md } \\
& \mathrm{K}_{\mathrm{m}} \quad=\text { matrix effective permeability, md } \\
& \mathrm{K}_{\mathrm{f}} \quad=\text { fracture intrinsic permeability tensor, md } \\
& \mathrm{k}_{\mathrm{B}}=\text { Boltzmann constant, } 1.38065 \times 10^{-23} \mathrm{~J} / \mathrm{K} \\
& \mathrm{K}_{\mathrm{n}} \quad=\text { Knudsen number } \\
& \mathrm{L}_{\mathrm{x}} \quad=\text { fracture spacing in } \mathrm{x} \text {-direction, } \mathrm{m} \\
& \mathrm{L}_{\mathrm{y}} \quad=\text { fracture spacing in } \mathrm{y} \text {-direction, } \mathrm{m} \\
& \mathrm{L}_{\mathrm{z}} \quad=\text { fracture spacing in } \mathrm{z} \text {-direction, } \mathrm{m} \\
& \mathrm{M} \quad=\text { mass accumulation, } \mathrm{kg} / \mathrm{m}^{3} \\
& \mathrm{M}_{\mathrm{g}} \quad=\text { gas molecular weight, } \mathrm{kg} / \mathrm{mol} \\
& \mathrm{P}=\text { pressure, } \mathrm{Pa} \\
& \mathrm{P}_{\mathrm{bh}} \quad=\text { bottomhole pressure, } \mathrm{Pa} \\
& \mathrm{P}_{\mathrm{m}} \quad=\text { matrix pressure, } \mathrm{Pa} \\
& \mathrm{P}_{\mathrm{mi}} \quad=\text { initial matrix pressure, } \mathrm{Pa} \\
& \mathrm{P}_{\mathrm{f}} \quad=\text { fracture pressure, } \mathrm{Pa} \\
& \mathrm{P}_{\mathrm{fi}} \quad=\text { initial fracture pressure, } \mathrm{Pa} \\
& \mathrm{P}_{\mathrm{L}} \quad=\text { Langmuir gas pressure, } \mathrm{Pa} \\
& \mathrm{q}=\text { mass of gas adsorbed gas per solid volume, } \mathrm{kg} / \mathrm{m}^{3} \\
& \bar{q}=\text { sink } / \text { source, } \mathrm{kg} / \mathrm{m}^{3} / \mathrm{s} \\
& \mathrm{q}_{\mathrm{a}}=\text { standard volume of adsorbed gas per solid mass, } \mathrm{m}^{3} / \mathrm{kg} \\
& \mathrm{r}=\text { pore radius, } \mathrm{m} \\
& \mathrm{R}=\text { universal gas constant, } \mathrm{Pa} \cdot \mathrm{m}^{3} /(\mathrm{mol} \cdot \mathrm{K}) \\
& \mathrm{S}_{\mathrm{g}} \quad=\text { gas saturation } \\
& \mathrm{t}=\text { time, } \mathrm{s} \\
& \mathrm{T}=\text { temperature, } \mathrm{K} \\
& \mathrm{T}_{\mathrm{mf}}=\text { transfer term, } \mathrm{kg} / \mathrm{m}^{3} / \mathrm{s} \\
& \mathrm{u}=\text { velocity, } \mathrm{m} / \mathrm{s}
\end{aligned}
$$


$\mathrm{V}_{\mathrm{L}} \quad=$ Langmuir gas volume, $\mathrm{m}$

$\mathrm{V}_{\text {std }}=$ gas molar volume at standard condition, $\mathrm{m}^{3}$

$\mathrm{z}=$ gas compressibility factor

\section{Greek symbols}

$\beta=$ tangential momentum accommodation coefficient

$\theta \quad=$ angle of anisotropy, ${ }^{\circ}$

$\lambda \quad=$ gas mean free path, $\mathrm{m}$

$\mu_{\mathrm{g}} \quad=$ gas viscosity, Pas

$\mu_{\mathrm{g} 0} \quad=$ initial gas viscosity, Pas

$\rho_{\mathrm{g}} \quad=$ gas density, $\mathrm{kg} / \mathrm{m}^{3}$

$\rho_{\mathrm{s}} \quad=$ shale density, $\mathrm{kg} / \mathrm{m}^{3}$

$\tau \quad=$ tortuosity

$\sigma \quad=$ Shape factor, $1 / \mathrm{m}^{2}$

$\phi_{\mathrm{m}} \quad=$ matrix porosity

$\phi_{\mathrm{f}} \quad=$ fracture porosity

$\omega \quad=$ rarefaction coefficient

\section{Subscripts}

$\begin{array}{ll}\text { app } & =\text { apparent } \\ \mathrm{bh} & =\text { bottomhole } \\ \mathrm{f} & =\text { fracture } \\ \mathrm{g} & =\text { gas } \\ \mathrm{L} & =\text { Langmuir } \\ \mathrm{m} & =\text { matrix } \\ \mathrm{std} & =\text { standard }\end{array}$

\section{SI Metric Conversion Factors}

$\begin{array}{lllll}\mathrm{atm} & \times & 1.01325 & \mathrm{E} 05 & =\mathrm{Pa} \\ \mathrm{bbl} & \times & 1.589873 & \mathrm{E}-01 & =\mathrm{m}^{3} \\ \mathrm{cp} & \times & 1 & \mathrm{E}-03 & =\mathrm{Pa} \cdot \mathrm{s} \\ \mathrm{ft} & \times & 3.048 & \mathrm{E}-01 & =\mathrm{m} \\ \mathrm{ft}^{3} & \times & 2.831685 & \mathrm{E}-02 & =\mathrm{m}^{3} \\ \mathrm{lbm} & \times & 4.5359 & \mathrm{E}-01 & =\mathrm{Kg} \\ \mathrm{md} & \times & 9.869233 & \mathrm{E}-16 & =\mathrm{m}^{2} \\ \mathrm{psi} & \times & 6.894757 & \mathrm{E} 00 & =\mathrm{kPa}\end{array}$

\section{References}

Aavatsmark, I., Barkve T., and Mannseth, T. 1998. Control-Volume Discretization Methods for 3D Quadrilateral Grids in Inhomogeneous, Anisotropic Reservoirs. SPE Journal 3 (2): 146-154. SPE-38000-PA. 10.2118/38000-PA.

Aavatsmark, I. 2002. An Introduction to Multipoint Flux Approximations for Quadrilateral Grids. Computational Geosciences 6 (3-4): 405-432. 
Aavatsmark, I. and Eigestad, G.T. 2006. Numerical Convergence of the MPFA O-Method and UMethod for General Quadrilateral Grids. International Journal for Numerical Methods in Fluids 51: 939-961.

Aavatsmark, I., Eigestad, G.T., and Klausen, R.A. 2006. Numerical Convergence of the MPFA OMethod for General Quadrilateral Grids in Two and Three Dimensions. Compatible Spatial Discretizations, The IMA Volumes in Mathematics and its Applications 142: 1-21.

Aavatsmark, I., Eigestad G.T., Klausen, R.A. et alet al. 2007. Convergence of A Symmetric MPFA Method on Quadrilateral Grids. Computational Geosciences 11: 333-345.

Aavatsmark, I., Eigestad, G.T., Mallison, B.T. et alet al. 2008. A Compact Multipoint Flux Approximation with Improved Robustness. Numerical Methods for Partial Differential Equations 24: 1329-1360.

Agelas, L. and Masson, R. 2008. Convergence of the Finite Volume MPFA O Scheme for Heterogeneous Anisotropic Diffusion Problems on General Meshes. Comptes Rendus Mathematique 346: 1007-1012.

Ayan, C., Colley, N., Cowan, G. et alet al. 1994. Measuring Permeability Anisotropy: The Latest Approach. Oilfield Review, Schlumberger 6: 24-35.

Barenblatt, G.E., Zheltov, I.P., and Kochina, I.N. 1960. Basic Concepts in the Theory of Seepage of Homogeneous Liquids in Fissured Rocks. Journal of Applied Mathematics and Mechanics 24: $1286-1303$.

Beskok, A. and Karniadakis, G.E. 1999. A Model for Flows in Channels, Pipes, and Ducts at Micro and Nano Scales. Microscale Thermophysical Engineering 3: 43-77.

Burton, D. Geologically-Based Permeability Anisotropy Estimates for Tidally-Influenced Reservoir Analogs using Lidar-Derived, Quantitative Shale Character Data. PhD Dissertation, The University of Texas at Austin, Austin, Texas (May 2011).

Cao, Y., Helmig, R., and Wohlmuth, B. 2009. Geometrical Interpretation of the Multipoint Flux Approximation L-Method. International Journal for Numerical Methods in Fluids 60: 1173-1199.

Civan, F., Rai, C.S., and Sondergeld, C.H. 2011. Shale-Gas Permeability and Diffusivity Inferred by Improved Formulation of Relevant Retention and Transport Mechanisms. Transport in Porous Media 86 (3): 925-944.

Cui, X., Bustin, A.M.M., and Bustin, R.M. 2009. Measurements of Gas Permeability and Diffusivity of Tight Reservoir Rocks: Different Approaches and Their Applications. Geofluids 9 (3): $208-$ 223.

Ding, D.Y., Wu, Y.S., Farah, N. et alet al. 2014. Numerical Simulation of Low Permeability Unconventional Gas Reservoirs. Presented at the SPE/EAGE European Unconventional Conference and Exhibition, Vienna, Austria, 25-27 February. SPE-167711-MS. 10.2118/167711-MS.

Edwards, M.G. and Rogers, C.F. 1998. Finite Volume Discretization with Imposed Flux Continuity for the General Tensor Pressure Equation. Computational Geosciences 2: 259-290.

Eigestad, G.T. and Klausen, R.A. 2005. On the Convergence of the Multi-point Flux Approximation O- Method: Numerical Experiments for Discontinuous Permeability. Numerical Methods for Partial Differential Equations 21: 1079-1098.

Fanchi, J. R. 2008. Directional permeability. SPE Reservoir Evaluation \& Engineering 11 (3): 565-568. 10.2118/102343-PA.

Guo, C., Wei, M., Chen, H. et alet al. 2014. Improved Numerical Simulation for Shale Gas Reservoirs. Presented at the Offshore Technology Conference-Asia, Kuala Lumpur, Malaysia, 25-28 March. OTC-24913-MS. 10.4043/24913-MS.

Heidemann, R.A., Jeje, A.A., and Mohtadi, F. An Introduction to the Properties of Fluids and Solids. University of Calgary Press. 
International Energy Agency. 2011. Are We Entering A Golden of Gas?. World Energy Outlook. Special Report.

Javadpour, F., Fisher, D., and Unsworth, M. 2007. Nanoscale Gas Flow in Shale Gas Sediments. Journal of Canadian Petroleum Technology 46 (10): 55-61. 10.2118/07-10-06.

Javadpour, F. 2009. Nanopores and Apparent Permeability of Gas Flow in Mudrocks. Journal of Canadian Petroleum Technology 48 (8): 16-21. 10.2118/09-08-16-DA.

Kazemi, H., Merrill, Jr., L.S., Porterfield, K.L. et alet al. 1976. Numerical Simulation of Water-Oil Flow in Naturally Fractured Reservoirs. SPE Journal 16 (6): 317-326. SPE-5719-PA. 10.2118/ 5719-PA.

Keilegavlen, E., Nordbotten, J.M., and Stephansen, A.F. 2012. Tensor Relative Permeabilities: Origins, Modeling, and Numerical Discretization. International Journal of Numerical Analysis and Modeling 9 (3): 701-724.

Klausen, R.A. and Winther, R. 2006. Robust Convergence of Multipoint Flux Approximation on Rough Grids. Numerische Mathematik 104: 317-337.

Klinkenberg, L.J. 1941. The permeability of Porous Media to Liquids and Gases. Drilling and Production Practice, New York, USA, 1 January. API-41-200.

Knudsen, M. 1909. Die gesetze der molekularstromung und der inneren reibungsstromung der gase durch rohren (The laws of molecular and viscous flow of gases through tubes). Annalen der Physik 333: $75-130$.

Kwon, O., Kronenberg, A.K., Gangi, A.F. et alet al. 2004. Permeability of Illite-Bearing Shale: 1. Anisotropy and Effects of Clay Content and Loading. Journal of Geophysical Research 109: B10205.

Langmuir, I. 1916. The Constitution and Fundamental Properties of Solids and Liquids. Journal of the American Chemical Society 38 (11): 2221-2295.

McCarthy, J.F. 1991. Analytical Models of the Effective Permeability of Sand-Shale Reservoirs. Geophysical Journal International 105 (2): 513-527.

Mehmani, A., Prodanovic, M., and Javadpour, F. 2013. Multiscale, Multiphysics Network Modeling of Shale Matrix Gas Flow. Transport in Porous Media 99 (2): 377-390.

Michalis, V.K., Kalarakis, A.N., Skouras, E.D. et alet al. 2010. Rarefaction Effects on Gas Viscosity in the Knudsen Transition Regime. Microfluidics and Nanofluidics 9: 847-853.

Negara, A., Salama, A., and Sun, S. 2013. 3-D Numerical Investigation of Subsurface Flow in Anisotropic Porous Media using Multipoint Flux Approximation Method. Presented at the SPE Reservoir Characterisation and Simulation Conference and Exhibition, Abu Dhabi, UAE, 16-18 September. SPE-165960-MS. 10.2118/165960-MS.

Negara, A., Salama, A., and Sun, S. 2014. Density-Driven Flow in Anisotropic Porous Media: Application to $\mathrm{CO}_{2}$ Geological Sequestration. Presented at the SPE Saudi Arabia Section Annual Technical Symposium and Exhibition, Al-Khobar, Saudi Arabia, 21-24 April. SPE-172232-MS. 10.2118/172232-MS.

Negara, A. Subsurface Flow Modeling in Single and Dual Continuum Anisotropic Porous Media using the Multipoint Flux Approximation Method.PhD Dissertation, King Abdullah University of Science and Technology, Thuwal, Saudi Arabia (May 2015).

Negara, A., Salama, A., and Sun, S. 2015. Multiphase flow simulation with gravity effect in anisotropic porous media using multipoint flux approximation. Computers \& Fluids 114: 66-74.

Nordbotten, J. and Eigestad, G.T. 2005. Discretization on Quadrilateral Grids with Improved Monotonicity Properties. Journal of Computational Physics 203: 744-760. 
Osman, H., Salama, A., Sun, S. et alet al. 2012. A Finite Difference, Multipoint Flux Numerical Approach to Flow in Porous Media: Numerical Examples. Presented at the Porous Media and Its Applications in Science, Engineering, and Industry: 4th International Conference, Potsdam, Germany, 17-22 June. AIP Conference Proceeding 1453: 217-222.

Ozkan, E., Raghavan, R., and Apaydin, O.G. 2010. Modeling of Fluid Transfer from Shale Matrix to Fracture Network. Presented at the SPE Annual Technical Conference and Exhibition, Florence, Italy, 19-22 September. SPE-134830-MS. 10.2118/134830-MS.

Pan, Z. and Connel, L.D. 2015. Reservoir Simulation of Free and Adsorbed Gas Production from Shale. Journal of Natural Gas Science and Engineering 22: 359-370.

Roy, S., Raju, R., Chuang, H.F. et alet al. 2003. Modeling Gas Flow through Microchannels and Nanopores. Journal of Applied Physics 93: 4870-4879.

Salama, A., Sun, S., and El Amin, M.F. 2013. A Multipoint Flux Approximation of the Steady-State Heat Conduction Equation in Anisotropic Media. ASME Journal of Heat Transfer 135:041302.

Salama, A., Sun, S., and Wheeler, M.F. 2014. Solving Global Problem by Considering Multitude of Local Problems: Application to Fluid Flow in Anisotropic Porous Media using the Multipoint Flux Approximation. Journal of Computational and Applied Mathematics 267: 117-130.

Salama, A., Negara, A., El Amin, M.F. et alet al. In press. Numerical investigation of nanoparticles transport in anisotropic porous media. Journal of Contaminant Hydrology (submitted 30 November 2014).

Shi, J., Zhang, L., Li, Y. et alet al. 2013. Diffusion and Flow Mechanisms of Shale Gas through Matrix Pores and Gas Production Forecasting. Presented at the SPE Unconventional Resources Conference-Canada, Calgary, Alberta, Canada, 5-7 November. SPE-167226-MS. 10.2118/167226-MS.

Sun, S., Salama, A., and El-Amin, M.F. 2012. An Equation-Type Approach for the Numerical Solution of the Partial Differential Equations Governing Transport Phenomena in Porous Media. International Conference on Computational Science, Procedia Computer Science 9: 661-669.

U.S. Energy Information Administration. 2013. Technically Recoverable Shale Oil and Shale Gas Resources: An Assessment of 137 Shale Formations in 41 Countries Outside the United States. Technical Report.

Warren, J.E. and Root, P.J. 1963. The Behavior of Naturally Fractured Reservoirs. SPE Journal 3 (3): 245-255. SPE-426-PA. 10.2118/426-PA.

Wu, Y.S., Li, J., Ding, D.Y. et alet al. 2014. A Generalized Framework Model for the Simulation of Gas Production in Unconventional Gas Reservoirs. SPE Journal 19 (5): 845-857. SPE-163609PA. 10.2118/163609-PA.

Xiong, X., Devegowda, D., Michel, G.G. et alet al. 2012. A Fully-Coupled Free and Adsorptive Phase Transport Model for Shale Gas Reservoirs including Non-Darcy Flow Effects. Presented at the SPE Annual Technical Conference and Exhibition, San Antonio, Texas, USA, 8-10 October. SPE-159758-MS. 10.2118/159758-MS. 